\title{
The impact of scarcity and abundance in food chains on species population dynamics*
}

\author{
Thomas Eichner and Rüdiger Pethig \\ University of Siegen \\ Department of Economics \\ Hoelderlinstr. 3 \\ D-57068 Siegen \\ Germany \\ phone: ++49 (0) $2717403164 / 3143$ \\ fax: $++49(0) 2717402732$ \\ e-mail: eichner@vwl.wiwi.uni-siegen.de \\ e-mail: pethig@vwl.wiwi.uni-siegen.de \\ forthcoming, Natural Resource Modeling
}

\begin{abstract}
The population dynamics in a food chain are derived from a sequence of short-run equilibria of an ecosystem where predator species demand prey biomass, supply own biomass to their predators and are assumed to behave as if they maximize net biomass intake. Introducing prices as scarcity indicators for the biomass of each species enables us to determine a short-run ecosystem equilibrium guided by prices. Equilibrium regimes differ with respect to their mix of zero-priced (= abundant) and positive-priced (= scarce) species. The population dynamics turn out to vary with the prevailing equilibrium regime. Our analysis yields a richer and more complex population dynamics than the traditional predator-prey dynamics of the Lotka-Volterra type.
\end{abstract}

*The authors gratefully acknowledge financial support by Deutsche Forschungsgemeinschaft. They are indebted to John Tschirhart and an anonymous referee for helpful and constructive comments. Remaining errors are the authors' sole responsibility. 


\section{Introduction}

Food chains are characterized by predator-prey interactions that are often modeled with the help of aggregate differential population equations of the Lotka-Volterra type. ${ }^{2}$ These authors provide a framework which is refined in different ways in the last decades. An example for recent developments are population equations with ratio-dependent functional responses (Berezovskaya, Karev and Ariditi 2001, Cantrell and Cosner 2001). Predatorprey systems of the Lotka-Volterra type provide valuable insights into the dynamics of interacting populations but their limitations have also been observed: Species interaction is assumed to be the interaction of populations in the sense that the growth of a given species does not only depend on its own population but also on the population of its predator species. But choosing populations as the basic endogenous variables amounts to disregarding the biomass transactions, and, more importantly, it fails to explain the types and scales of those transactions.

With the aim of providing a theoretical foundation for the population dynamics, we are lead by striking similarities between ecosystems and economic systems to model ecosystems by means of economic methodology: The first important distinction is between the short and the long term with populations being constant in the short-run while they vary in the long-run. In economic processes, capital stocks are fix in the short-run period but investment (net of depreciation) in that period makes capital accumulate or shrink in successive periods. The analogue of economic capital investment in ecosystems is the net biomass (number of offspring) a species acquires in the short-run period.

A further similarity between both systems is the relevance of transactions of commodities and biomass, respectively, which suggests to look at ecosystems in terms of supply of a prey's biomass to predators and of a predator's demand for prey biomass. We finally add prices as scarcity indicators for each type of biomass and introduce the assumption that all species behave as if they maximize their (short-run period) net biomass. With these ingredients, the ecosystem agents' optimal demands and supplies can be thought of as being coordinated by prices. Thus, the concept of a general short-run ecosystem equilibrium is put forward which is equivalent, in spirit, to the notion of a general perfectly competitive economic equilibrium.

Implicitly, the equilibrium allocation of biomass transactions completely determines the net biomass (number of offspring) each species acquired during the short-run period. When combined with the populations that have prevailed and kept constant during that period, we end up with a system of differential population equations, one equation for

\footnotetext{
${ }^{2}$ The classical Lotka-Volterra model dates back to Lotka (1925) and Volterra (1926).
} 
each species. In other words, we derive the population dynamics from more basic assumptions of our model while, in contrast, differential population equations constitute the basic assumptions of Lotka-Volterra type models.

The methodology of modelling ecosystems as outlined above has been developed by Hannon (1976), Crocker and Tschirhart (1992), Pethig and Tschirhart (2001) and Tschirhart (2000, 2002), but the approach chosen here exhibits a number of differences that will be elucidated below. Our most substantial deviation from earlier work is to focus on satiation in the demand for zero-priced biomass. This assumption is clearly realistic because, during a short-run period, predators can hardly forage arbitrarily large amounts of prey biomass, irrespective of whether they face predation risks themselves or whether their prey species is abundant. Gurney and Nisbet (1998, p. 161) go on to point out that 'the functional response of all organisms must extremely saturate at an uptake rate matching the maximum rate at which ingestate can be processed'. While the satiation assumption is empirically relevant, it may be viewed, at the first glance, as a minor technical detail only. However, it will turn out to be highly consequential both regarding the complexity of the formal analysis and with respect to the resultant population dynamics.

To give a non-technical account of the problem at issue recall first from the economics of competitive equilibrium that when a market for a commodity is in equilibrium at price zero, demand matches supply or the commodity is in excess supply. Commodities in strict excess supply (free goods) are an equilibrium feature because no economic agent has any use for that excess supply. In contrast, the species that supplies own biomass is highly interested to 'appropriate' that excess supply since losing own biomass reduces own net biomass. Zero-priced prey biomass is therefore a free good for the predator (due to satiation) but not for the prey, and hence strict market clearing needs to be required irrespective of whether equilibrium prices are zero or positive.

The important implication of this observation is the impact of the mix of zero-priced and positive-priced species on the associated population dynamics. Clearly with three species and the possibility of each equilibrium price being positive or zero we obtain eight different equilibrium regimes each of which commands a specific set of three differential equations. Therefore the time path of growing or shrinking populations not only depends on the set of populations in the initial short-run period but also on the sequence of different equilibrium regimes the ecosystem passes in time. In other words, accounting for scarcity (positive price) and abundance (zero price) of biomass makes population dynamics in a food chain richer and more complex than those assumed in models of the Lotka-Volterra type. 
Section 2 outlines the model of the short-run period, discusses its basic premises and introduces the concept of general short-run ecosystem equilibrium. In Section 3 such equilibria are characterized in a parametric version of that model. Special emphasis is placed on how the set of species populations, assumed constant in the short-term, determines the abundance or scarcity of species in the pertaining short-run equilibrium. Section 4 derives and discusses the differential population equations implied by the model, one equation for each species, in short-run equilibrium regimes, demonstrating that all these differential equations are regime-specific. The numerical analysis of population growth of Section 5 exemplifies the time path of the population(s) of one or two species in a three-species food chain. For given populations of species 2 and 3 the population of species 1 is shown to induce (no, one or two) switches of equilibrium regimes and to exhibit a logistic growth pattern similar as assumed in Verhulst-Pearl type of models. When only the population of species 1 is kept constant, the remaining species grow in various ways (and may or may not switch regimes) until, eventually, they tend towards a joint steady state.

\section{The model of the short-run period}

Consider an ecosystem of fixed size where $m$ species form a one-directional non-circular food chain: Species $m$ feeds on species $m-1, \ldots$, species 2 feeds on species 1 , and species 1 feeds on a resource referred to as 'species 0' in the formal model. To simplify, we choose the aggregate species rather than the individual organisms of each species as the basic unit of analysis. ${ }^{3}$

Following Tschirhart (2000) we define the short-run as that time over which the populations of all species are constant. In a given short-run period, $x_{i-1}$ is species $i$ 's intake of biomass of species $i-1$ (demand), $s_{i}$ is species $i$ 's loss or sacrifice of own biomass ${ }^{4}$ (supply) to its predators, $h_{i}$ are species $i$ 's costs of searching for food, attacking and handling its prey, its hunting cost, for short, and $d_{i}$ is species $i$ 's death rate. For any given $\left(h_{i}, s_{i}, x_{i-1}, n_{i}\right)$

$$
\dot{n}_{i}:=\frac{\mathrm{d} n_{i}}{\mathrm{~d} t}=B^{i}\left(\begin{array}{c}
h_{i}, s_{i}, x_{i-1}, n_{i} \\
-{ }_{-}+d_{i}
\end{array}\right)-d_{i}
$$

is the net biomass acquired by species $i$ in the short-run period under consideration. In (1) the variables $h_{i}, s_{i}$ and $x_{i-1}$ and the net biomass $B^{i}$ are measured in biomass units (e.g. $\mathrm{kg}$ ) per unit of time $t$. $B^{i}$ is a concave function. The signs of its partial derivatives (indicated in (1) by plus or minus signs) are straightforward for $h_{i}, n_{i}$ and $s_{i}$. Regarding

\footnotetext{
${ }^{3}$ For more details, see footnote 9 below.

${ }^{4}$ For $i=1, x_{i-1}$ is the resource intake of species 1 and for $i=0, s_{0}=\bar{s}_{0}$ is the fixed ecosystem endowment of the resource.
} 
$x_{i-1}$, we assume $B_{x x}^{i}<0$ for all $x_{i-1} \geq 0, B_{x}^{i}>0$ for small $x_{i-1}$ and $B_{x}^{i}<0$ for large $x_{i-1}$. Clearly, species $i$ cannot expand its biomass indefinitely by arbitrarily large prey intake during any given period.

The function $B^{i}$ represents an aggregate physiological function similar to the one employed by Hannon (1976), Crocker and Tschirhart (1992) and Tschirhart (2000, 2002) at the level of individual organisms. Physiological functions account for maintenance, respiration and metabolism. From an economist's perspective, $B^{i}$ resembles a production function in that the inputs 'biomass of species $i-1$ ' and 'food searching effort' are transformed into the output 'biomass of species $i$ '. But rather than taking the entire output to the market for sale (as economic producers usually do), species $i$ offers only the amount $s_{i}$ of its 'product' for sale (and does so only reluctantly). The remaining biomass 'produced' is transfered to the next period (to be specified below). To put it differently, economic entrepreneurs use inventories mainly as a buffer against asynchronous production and sales. In contrast, species are preoccupied with piling up their 'inventory'.

The existence and the role of commodities and transactions is strikingly similar between ecosystems and economies. In ecosystems, the biomass of each species is a commodity and so are resources (nutrients, sunlight, water, etc.). The latter are used as primary inputs and the former as intermediate factors of biomass production. Transactions take place via predator-prey interactions which constitute intra-ecosystem trade. Traders either demand biomass of other species (predators) or supply own biomass (preys). The species at the top [bottom] of the food chain is a predator [prey] species only ${ }^{5}$ but all intermediate members of the food chain are both predators and preys. Such an intermediate species $i$ is prey for species $i+1$ and hence is bound to supply own biomass, $s_{i}$ (although it does so reluctantly only). The predator $i+1$, in turn, demands the amount $x_{i}$ of biomass of species $i$. Thus we have a commodity, namely the biomass of (prey) species $i$, and we have a supply of and a demand for that commodity. In economic models, these are the essential prerequisites for a market, which doesn't exist in the ecosystem, of course, in an institutionalized sense with exchanges of commodities against money. But, returning to the ecosystem, suppose $\hat{x}_{i}>0$ is species $i+1$ 's unconstrained most wanted intake of prey biomass (to be specified below as its 'satiation demand') and consider a situation of excess demand: $\hat{x}_{i}>s_{i}$. Intuitively speaking, species $i$ is then scarce and one needs to introduce an allocation mechanism capable to bring about the strict equality $\hat{x}_{i}=s_{i}$ which is necessary for an allocation to be feasible. In economic models the problem of matching demand and supply is solved by the price mechanism: Competition on the part of demanders (in

\footnotetext{
${ }^{5}$ In real ecosystems top predators do die and decay and are eaten by the decomposers. In that sense they too are prey. This circular flow aspect of the food chain is neglected here to keep the model tractable.
} 
case of excess demand) drives the price up until total demand equals total supply. Similarly, if $\hat{x}_{i}>s_{i}$ then predator organisms need to compete for prey biomass. We will assume that all species receive information about scarcities in form of prices that impact on their opportunity sets and hence on their supplies and demands. As in economic models, our ecosystem prices have the ultimate function of equating the demand for and the supply of all species' biomass. Yet with this approach we need not and do not subscribe to the notion of an ecosystem consisting of institutionalized markets and real biomass sales and purchases for (fiat) money. ${ }^{6}$

To be more specific, denote by $p:=\left(p_{0}, p_{1}, \ldots, p_{m-1}\right) \in \mathbb{R}_{+}^{m}$ a vector of prices such that $p_{i}$ is the price of biomass of species $i=0,1, \ldots, m-1$. Prices are signals of scarcity $\left(p_{i}>0\right)$ or abundance $\left(p_{i}=0\right)$ and these signals are assumed to be perceived by all species. ${ }^{7}$ Each species takes as given the price signals and responds to them in a way to be specified below. On the other hand, all prices are endogenous to the ecosystem as a whole and adjust until there is a perfect match of demand for and supply of biomass of all species.

The next step is to introduce hypotheses about the costs of searching for food, $h_{i}$, and the supply of own biomass, $s_{i}$ :

$$
\begin{array}{cc}
h_{i}=H^{i}\left(\begin{array}{c}
p_{i-1}, x_{i-1} \\
\left.+\begin{array}{c}
+
\end{array}\right)
\end{array}\right. & \text { for } \quad i=1, \ldots, m, \\
s_{i}=\min \left[\begin{array}{c}
\bar{x}_{i}, R^{i}\left(\begin{array}{c}
\left.p_{i}, x_{i-1}\right) \\
+
\end{array}\right] \\
+
\end{array}\right] \quad \text { for } \quad i=1, \ldots, m-1 .
\end{array}
$$

The assumptions $H_{x}^{i}>0$ and $H_{p}^{i}>0$ are motivated by the considerations that capturing more prey (i.e. increasing $x_{i-1}$ ) involves greater costs of searching for food and that the effort of capturing a given amount of prey must be stepped up, ceteribus paribus, with increasing price $p_{i-1}$, see also Stephens and Krebs (1986). The way $p_{i-1}$ affects the hunting costs (2) is indicative of the role of prices in the ecosystem, in general. (2) is meant to express the hypothesis that for a given intake $x_{i-1}$ the hunting costs species $i$ incurs are the greater the scarcer the biomass of species $i$ is. Scarcity of biomass $i$ is measured in (2) by $p_{i-1}$ which is perceived and taken as given by all species $i$ (as well as by all other species) during the period under consideration. As a consequence, species $i$ 's optimal demand-supply plan (to be specified below) depends on the vector $p \in \mathbb{R}_{+}^{m}$ of scarcity

\footnotetext{
${ }^{6}$ It is interesting to note that the standard neoclassical economic allocation theory also describes a world without institutionalized markets and (fiat) money, if it is stripped off all (economic) interpretations that are not essential for its formal structure.

${ }^{7}$ The assumption is certainly as unrealistic as the often criticized assumption of market transparency in (most) economic models.
} 
indicators. For given $p_{i}>0$, the hypothesis $R_{x}^{i}>0$ reflects species $i$ 's risk of being preyed on while foraging. This risk is known as the predation risk of species $i$ (Lima and Dik 1990). Foraging takes time and requires exposure to predators. The more prey is taken in by species $i$, i.e. the greater is $x_{i-1}$, the greater is the (expected) loss of own biomass, $s_{i}$. $R_{p}^{i}>0$ expresses the view that the predation risk of species $i$ also increases with species $i$ 's own scarcity: The rational behind this hypothesis is that $p_{i}$ rises when the predator species $i$ steps up its demand for biomass $i$, and that the prey species $i$ cannot help but yield, to some extent at least, to its predator's increasing demand pressure. Obviously, the highest-ranking predator species does not face any predation risk since it lacks natural enemies; hence $s_{m} \equiv 0$.

The equations (2) and (3) can be interpreted as expressing the view that $h_{i}$ and $s_{i}$ together are species $i$ 's "total price" to be paid for the intake $x_{i-1}$ of prey biomass. The costs $h_{i}$ are lost to the ecosystem (e.g. energy burnt up by $i$ ) while $s_{i}$ represents a transfer from species $i$ to species $i+1$. As specified in (2) and (3) prices do have an impact on $h_{i}$ and $s_{i}$ with marginal contributions $H_{p}^{i}>0$ and $R_{p}^{i}>0$ (ignoring $\bar{x}_{i}$ ) respectively. But as noted before, prices are not something that predators pay for their consumption of prey biomass in a market place and that the prey receives as a compensation for sacrifying own biomass. Nonetheless, we consider it appropriate in the present context to refer to the vector $p$ as prices, because $p$ is endogenous to the model and serves the central function of equilibrating all demands and supplies (to be formalized below).

Having clarified the "total price" predator $i$ has to pay for $x_{i-1}$ let us also ask what the prey gets in return. The prey $i-1$ doesn't receive any benefit from its predator species $i$ but replacing $i$ by $i-1$ and $i-1$ by $i-2$ in (2) and (3) reveals that prey $i-1$ doesn't offer any own biomass $s_{i-1}>0$ and doesn't incur any costs $h_{i-1}$ unless $x_{i-2}>0$ in the first place. In other words, by offering $s_{i-1}>0$ it acquires the right to a positive amount $x_{i-2}$ of biomass of species $i-2$. Species $i-1$ is interested in $x_{i-2}$ only but for taking in $x_{i-2}>0$ it has to pay the "total price" consisting of $h_{i-1}$ and $s_{i-1}$. Obviously all intermediate members of the food chain are in a perfectly symmetric position.

The min $[\cdot, \cdot]$ specification in $(3)$ has no counterpart in models of competitive economies and therefore calls for a careful foundation. $\bar{x}_{i}$ is an upper bound or constraint on the supply of biomass of species $i$. This constraint is exogenous from the viewpoint of species $i$ but will be made endogenous to the model (as clarified in the equilibrium definition below) in such a way that it is binding if and only if $p_{i}=0$. In other words, the min $[\cdot, \cdot]$ term is designed to prevent markets for biomass to exhibit an excess supply at zero price. As mentioned above, in competitive economies situations of strict excess supply at zero prices qualify as equilibrium characterizing the respective commodity as a free good that is of no 
use to any agent. If an ecosystem equilibrium would exhibit an excess supply of some prey species at price zero, this excess supply is not of any use for its predator species, yet the prey species does appreciate - and is happy about not losing - this 'excess supply'. It only loses the amount $\min \left[R^{i}\left(p_{i}=0, x_{i-1}\right), \bar{x}_{i}\right]$ of own biomass as required in (3).

In view of (1), (2) and (3) the net biomass $\dot{n}_{i}$ from (1) that species $i$ acquires in the short-run period under consideration is completely determined by species $i$ 's choice of $s_{i}$ and $x_{i-1}$ for given $\bar{x}, p \in \mathbb{R}_{+}^{m-1}$. (Note that $s_{i}$ depends on $x_{i-1}$ via $(3)$ and therefore $x_{i-1}$ is species $i$ 's only effective choice variable). The species' decision making problem is assumed to be as follows: For any given $\bar{x}$ and $p$, species $i$ behaves as if it maximizes (1) subject to (2) and (3). ${ }^{8}$ Clearly, the maximizers $x_{i-1}$ and $s_{i}$ depend on $p, n$ and $\bar{x}$. Hence solving the maximization problem yields demand functions $X^{i-1}(p, \bar{x}, n)$ for $i=1, \ldots, m$ and supply functions $S^{i}(p, \bar{x}, n)$ for $i=1, \ldots, m-1$.

As in case of a perfectly competitive economy we are interested in a situation in which all markets for biomass clear. More specifically, an ecosystem allocation of transactions $\left(x^{*}, s^{*}\right)$, prices $p^{*}$ and constraints $\bar{x}$ is said to constitute a general short-run ecosystem equilibrium, if for given populations $n$,

$$
\begin{array}{rlrl}
x_{i}^{*}=X^{i}\left(p^{*}, \bar{x}, n\right) & & i=0,1, \ldots, m-1, \\
s_{i}^{*}=S^{i}\left(p^{*}, \bar{x}, n\right) & & i=1, \ldots, m-1, \\
\bar{s}_{0} \geq x_{0}^{*} \text { and } p_{0}^{*}\left(\bar{s}_{0}-x_{0}^{*}\right)=0, & \\
s_{i}^{*}=x_{i}^{*} & & i=1, \ldots, m-1, \\
\bar{x}_{i}=X^{i}\left(p_{-i}^{*}, \bar{x}, n\right) & & i=1, \ldots, m-1,
\end{array}
$$

where $p_{-i}^{*}:=\left(p_{0}^{*}, \ldots, p_{i-1}^{*}, 0, p_{i+1}^{*}, \ldots, p_{m-1}^{*}\right)$.

Recall from our discussion of equation (3) above that there is a major difference between this notion of equilibrium and the equilibrium in perfectly competitive economies: If in equilibrium the biomass of some species commands the price zero the market does not exhibit an excess supply because we require the (strict) equality sign in (4d), which implies, in turn, that a zero-priced biomass is not a 'free good' in the same sense as zero-priced economic commodities are. Observe, however, that due to $(4 \mathrm{c})$ the resource $(i=0)$ may be zero-priced and in (strict) excess supply in an ecosystem equilibrium just like zero-priced

\footnotetext{
${ }^{8}$ The assumption of optimizing behavior of individual organisms has a long tradition in evolutionary ecology, see Houston and McNamara (1999). To our knowledge, in the context of formal analysis of the short-run period of ecosystems it has first been suggested and employed by Hannon (1976) and has since been used in several other studies, e.g. in Crocker and Tschirhart (1992), Tschirhart (2000, 2002) and Pethig and Tschirhart (2001).
} 
economic commodities in perfectly competitive equilibrium. For convenience we refer to the zero-priced resource as well as to zero-priced biomass as being abundant. ${ }^{9}$

As shown in Appendix A (claim A3) the equilibrium condition (4e) sees to it that the supply constraints are binding $\left(s_{i}^{*}=\bar{x}_{i}\right)$ if and only if species $i$ is abundant.

\section{Characterization of short-run ecosystem equilibria in a parametric model}

Even though our model of the short-run period is quite simple it is hardly possible to obtain further specific information about the characteristics of a short-run ecosystem equilibrium unless more structure is placed on the functions $B^{i}, H^{i}$ and $R^{i}$ from (1) - (3). Therefore we proceed by employing the following parametric functions:

$$
\begin{aligned}
B^{i}\left(h_{i}, s_{i}, x_{i-1}, n_{i}\right) & =\left(a_{i} n_{i}-\frac{c_{i} x_{i-1}}{2}\right) x_{i-1}-h_{i}-s_{i}, \\
H^{i}\left(p_{i-1}, x_{i-1}\right) & =\left(e_{i}+f_{i} p_{i-1}\right) x_{i-1} \\
R^{i}\left(p_{i}, x_{i-1}\right) & =g_{i} p_{i}+k_{i} x_{i-1}
\end{aligned}
$$

where $a_{i}, c_{i}, e_{i}, f_{i}, g_{i}, k_{i}$ are positive parameters for $i=1,2,3$ except for $g_{3}=k_{3}=0$.

\section{Proposition 1.}

Define $\tilde{x}_{i-1}:=\frac{a_{i} n_{i}-e_{i}-f_{i} p_{i-1}}{c_{i}}, \hat{x}_{i-1}:=\frac{a_{i} n_{i}-e_{i}-k_{i}-f_{i} p_{i-1}}{c_{i}}, \hat{r}_{i}:=g_{i} p_{i}+k_{i} \hat{x}_{i-1}$, and $\bar{X}^{i}\left(p_{i}, p_{i-1}\right):=\frac{2 k_{i}\left(a_{i} n_{i}-e_{i}-f_{i} p_{i-1}\right)-k_{i}^{2}-2 c_{i} g_{i} p_{i}}{2 c_{i}}$.

For any given vectors $p$ and $\bar{x}$ : Maximizing (1) subject to (3), (5a) - (5c) yields:

For $i=1,2$ :

$$
\left(s_{i}^{*}, x_{i-1}^{*}\right)=\left\{\begin{array}{c}
\left(\bar{x}_{i}, \tilde{x}_{i-1}\right) \\
\left(\bar{x}_{i}, \tilde{x}_{i-1}\right) \text { and }\left(\hat{r}_{i}, \hat{x}_{i-1}\right) \\
\left(\hat{r}_{i}, \hat{x}_{i-1}\right)
\end{array}\right\} \Longleftrightarrow \bar{x}_{i}\left\{\begin{array}{c}
< \\
= \\
>
\end{array}\right\} \bar{X}^{i}\left(p_{i}, p_{i-1}\right) .
$$

\footnotetext{
${ }^{9}$ Another remark on the definition of equilibrium is in order. In the model of the present paper we choose species rather than individual organisms as the basic units of analysis. A more ambitious and more appealing approach would provide a microfoundation by taking representative individual organisms of each species as the basic units of analysis. This route is taken, in fact, by Tschirhart (2000). To see the consequences of this alternative approach for defining equilibrium, suppose for the moment that the index $i$ in our model indicates the representative individual organism of species $i$ rather than species $i$ itself. Then the most consequential change would be to replace (4d) by the equations $n_{i} s_{i}^{*}=n_{i+1} x_{i}^{*}$. (At the same time one might want to drop $n_{i}$ as an argument of function $B^{i}$ from (1)). The only reason why we refrain from this approach is to keep the analysis tractable. Observe that for similar reasons large-scale models of an economy take industries or branches as their basic decision units rather than individual firms.
} 
For $i=3$ we obtain $\left(s_{2}^{*}, x_{2}^{*}\right)=\left(0, \hat{x}_{2}\right)$.

Proposition 1 is proved in Appendix A. It provides the solution to all species' maximization problems depending on whether the supply constraint $\bar{x}_{i}$ is strictly binding $\left(\bar{x}_{i}<\bar{X}^{i}(\cdot)\right)$, weakly binding $\left(\bar{x}_{i}=\bar{X}^{i}(\cdot)\right)$ or not binding at all $\left(\bar{x}_{i}>\bar{X}^{i}(\cdot)\right)$. Observe that the demand functions $X^{i}$ are single-valued and smooth except when the supply constraint is weakly binding.

We already indicated in our discussion of equation (3) above that the supply constraints $\bar{x}$ are designed to rule out an excess supply of zero-priced biomass in equilibrium. As shown in Appendix A, this goal is in fact achieved:

\section{Proposition 2.}

Suppose, $\left(p, s^{*}, x^{*}, \bar{x}\right)$ is a short-run ecosystem equilibrium.

$$
p_{i}\left\{\begin{array}{l}
= \\
>
\end{array}\right\} 0 \quad \Longleftrightarrow \quad \bar{x}_{i}\left\{\begin{array}{c}
\leq \\
>
\end{array}\right\} \bar{X}^{i}\left(p_{i}, p_{i-1}\right) .
$$

Propositions 1 and 2 combined with the definition of equilibrium $((4 a)-(4 e))$ enable us to completely determine a short-run equilibrium. Clearly, the characteristics of such an equilibrium depend heavily on equilibrium prices being positive or zero. As shown in Table 1, eight equilibrium regimes need to be distinguished, where an entry "0" stands for biomass abundance $\left(p_{i}=0\right)$ while " 1 " indicates biomass scarcity $\left(p_{i}>0\right)$. Recall that in the short-run period the populations $n_{i}$ of all species $i=1,2,3$ are constant. Moreover, demand and supply functions depend on populations (as can be seen from Proposition 1). Hence prices designed to equilibrate supply and demand are bound to depend on the size of populations, too. This observation suggests that, ceteris paribus, a prey species $i$ is the more likely to be scarce $\left(p_{i}>0\right)$, the greater is the population of its predator, i.e. the larger is $n_{i+1}$.

Next we will show that for each regime $j, j=1, \ldots, 8$, there is a non-empty support set $N_{j} \subset \mathbb{R}_{+}^{3}$ with the following property: The equilibrium regime $j$ emerges if and only if the populations prevailing in the short-run period under consideration satisfy $\left(n_{1}, n_{2}, n_{3}\right) \in$ $N_{j}$. In fact, all support sets $N_{j}$ are disjoint. 


\begin{tabular}{|c|l|l|l|l|l|l|l|l|l|}
\hline $\operatorname{sign} p$ & Regime No. & 1 & 2 & 3 & 4 & 5 & 6 & 7 & 8 \\
\hline \hline$p_{0}$ & 0 & 0 & 0 & 0 & 1 & 1 & 1 & 1 \\
\hline$p_{1}$ & 0 & 0 & 1 & 1 & 1 & 0 & 0 & 1 \\
\hline$p_{2}$ & 0 & 1 & 1 & 0 & 0 & 0 & 1 & 1 \\
\hline
\end{tabular}

Table 1: Feasible equilibrium regimes

To determine the support sets we need to combine the Propositions 1 and 2 with the definition of equilibrium. We will not work through all eight equilibrium regimes but content ourselves, instead, with demonstrating the procedure by focussing on the polar regimes 1 and 8 .

Regime 1. Invoking (4e) for $i=0,(4 \mathrm{c})$ and $x_{0}^{*}=\tilde{x}_{0}$ for $p_{0}=0$ (from Propositions 1 and 2) we conclude that in equilibrium the market for the resource $(i=0)$ satisfies

$$
\bar{s}_{0} \geq \frac{a_{1} n_{1}-e_{1}}{c_{1}}, \quad \text { or, equivalently, } \quad n_{1} \leq \frac{e_{1}+c_{1} \bar{s}_{0}}{a_{1}} .
$$

We also know from Proposition 2 that setting $p_{i}=0$ for $i=1,2$ implies

$$
\begin{gathered}
\frac{a_{i+1} n_{i+1}-e_{i+1}}{c_{i+1}} \leq k_{i}\left(\frac{a_{i} n_{i}-e_{i}}{c_{i}}-\frac{k_{i}}{2 c_{i}}\right) \quad \text { and hence } \\
n_{i+1} \leq \frac{a_{i} c_{i+1} k_{i}}{a_{i+1} c_{i}} n_{i}+\frac{2 c_{i} e_{i}-c_{i+1} k_{i}\left(2 e_{i}+k_{i}\right)}{2 a_{i+1} c_{i}}, \quad i=1,2 .
\end{gathered}
$$

Therefore the short-run ecosystem equilibrium belongs to regime 1 , if and only if

$$
\left(n_{1}, n_{2}, n_{3}\right) \in N_{1}:=\left\{\left(n_{1}, n_{2}, n_{3}\right) \mid(6) \text { and }(7) \text { are satisfied }\right\} .
$$

In such an equilibrium the transactions are

$$
s_{i-1}^{*}=x_{i-1}^{*}=\frac{a_{i} n_{i}-e_{i}}{c_{i}} \text { for } i=2,3 \quad \text { and } \quad x_{0}^{*}=\frac{a_{1} n_{1}-e_{1}}{c_{1}},
$$

and the associated prices are $\left(p_{0}, p_{1}, p_{2}\right)=(0,0,0)$. This information fully characterizes the equilibrium regime 1.

Regime 8. We know from Proposition 2 that owing to $p_{i}>0$ for $i=0,1,2$ the supply constraints $\bar{x}_{1}$ and $\bar{x}_{2}$ are not binding. Moreover, in equilibrium (4c) holds as an equality, and therefore $x_{i}^{*}=s_{i}^{*}$ for $i=0,1,2$. We apply the appropriate demand and supply functions from Proposition 1 and obtain, after some suitable rearrangements,

$$
\begin{aligned}
\frac{a_{1} n_{1}-e_{1}-k_{1}-f_{1} p_{0}}{c_{1}} & =\bar{s}_{0}, \\
\frac{a_{2} n_{2}-e_{2}-k_{2}-f_{2} p_{1}}{c_{2}} & =g_{1} p_{1}+k_{1} \bar{s}_{0}, \\
\frac{a_{3} n_{3}-e_{3}-f_{3} p_{2}}{c_{3}} & =g_{2} p_{2}+g_{1} k_{2} p_{1}+k_{1} k_{2} \bar{s}_{0} .
\end{aligned}
$$


These equations jointly determine the equilibrium price vector $\left(p_{0}, p_{1}, p_{2}\right)$ :

$$
\begin{aligned}
& p_{0}=\frac{a_{1} n_{1}-e_{1}-k_{1}-c_{1} \bar{s}_{0}}{f_{1}}, \\
& p_{1}=\frac{a_{2} n_{2}-e_{2}-k_{2}-c_{2} k_{1} \bar{s}_{0}}{c_{2} g_{1}+f_{2}}, \\
& p_{2}=\frac{a_{3} n_{3}-e_{3}-c_{3} k_{1} k_{2} \bar{s}_{0}-\frac{c_{3} g_{1} k_{2}}{c_{2} g_{1}+f_{2}}\left(a_{2} n_{2}-e_{2}-k_{2}-c_{2} k_{1} \bar{s}_{0}\right)}{c_{3} g_{2}+f_{3}} .
\end{aligned}
$$

So far we calculated the equilibrium prices in (11a) - (11c) but we haven't yet specified $N_{8}$, the support set of regime 8 . For defining $N_{1}$ we made use of the conditions $\bar{x}_{i} \leq \bar{X}\left(p_{i}, p_{i-1}\right)$, $i=1,2$, from Proposition 2. In case of $N_{8}$ the analogous procedure would call for employing the inequalities $\bar{x}_{i}>\bar{X}\left(p_{i}, p_{i-1}\right)$ for $i=1,2$ which amounts to placing rather complex constraints on $\left(n_{1}, n_{2}, n_{3}\right)$. Note, however, that according to Proposition $2 \bar{x}_{i}>\bar{X}\left(p_{i}, p_{i-1}\right)$ is equivalent to $p_{i}>0$. Hence we take the less complex route and safely define

$$
N_{8}:=\left\{\left(n_{1}, n_{2}, n_{3}\right) \mid(11 \mathrm{a})-(11 \mathrm{c}) \text { holds and } p_{i}>0 \text { for } i=0,1,2 .\right\} .
$$

The transactions associated to an equilibrium of regime 8 are calculated by inserting prices (11) into (10):

$$
\begin{aligned}
x_{0}^{*} & =\bar{s}_{0}^{*}, \\
x_{1}^{*} & =\frac{g_{1}\left(a_{2} n_{2}-e_{2}-k_{2}\right)+f_{2} k_{1} \bar{s}_{0}}{c_{2} g_{1}+f_{2}} \\
x_{2}^{*} & =\frac{\left(c_{2} g_{1}+f_{2}\right)\left[f_{3} k_{1} k_{2} \bar{s}_{0}+\left(a_{3} n_{3}-e_{3}\right) g_{2}\right]+g_{1} k_{2}\left(a_{2} n_{2}-e_{2}-k_{2}-c_{2} k_{1} \bar{s}_{0}\right)}{\left(c_{2} g_{1}+f_{2}\right)\left(c_{3} g_{2}+f_{3}\right)} .
\end{aligned}
$$

The equilibrium prices (11) and the transactions (13) deserve a comment: Since the ecosystem under consideration is a one-directional non-circular food chain, any exogenous shock to the ecosystem (in form of a change of some parameter) has an impact upstream in the chain only, ${ }^{10}$ since $p_{i}$ and $x_{i}^{*}$ in (11) and (13) contain parameters with subscript $j=0, \ldots, i-1$ but not with subscripts $j>i$. Consequently, the higher a species' rank in the food chain the more it is exposed to downstream shocks, the more its scarcity and the volume of own biomass transactions depends on what happens downstream.

Quite obviously, our model allows for a rich comparative static analysis of parameter shocks which in turn generate a great variety of testable hyptheses. ${ }^{11}$ In applied case studies it is both important and worthwhile to determine and to test the impact of individual parameter changes on the ecosystem allocation. The discussion of such issues is beyond

\footnotetext{
${ }^{10}$ This is an example of a bottom up food chain which in the biological literature is also called donor controlled, compare Pimm (1982).

${ }^{11}$ The complexity of (11) and (13) suggests, however, that the net effects of parameter shifts will not always be unambiguous in sign.
} 
the scope of the present paper. Yet we want to briefly indicate the type of comparative static information contained in (11) and (13) by means of a few examples. An expansion of the ecosystem's resource base (an exogeneous increase in $\bar{s}_{0}$ ) reduces the scarcity of species 0 and 1 and boosts the market transactions of their biomass. An increase in $\bar{s}_{0}$ renders species 2 also less scarce but $x_{2}^{*}$ may rise or fall. An exogenous increase in $n_{i}$ caused, e.g., by immigration intensifies the pressure put on prey species $i-1$ by its predator $i$ to the effect that the price of prey biomass $i-1$ rises for $i=1,2,3$ and the associated transactions rise, too, for $i=2,3$. An interesting additional effect can be observed with regard to the biomass of species 2. Its increasing scarcity upon an exogenous rise in $n_{3}$ tends to be compensated, if there is a simultaneous exogenous increase in species 2's own population which, on the other hand, further expands transactions $x_{2}^{*}$. These effects of exogenous shocks in $n_{2}$ on the market for biomass 2 appear to be in line with one's intuition. Their origin is clearly the positive price elasticity of supply of biomass of species $i\left(g_{i}>0\right)$. Since by assumption the price elasticity of the resource supply is zero, these effects are missing in case of species 1 .

Since the remaining regimes $2, \ldots, 7$ are intermediate cases of the regimes 1 and 8 , the methodology of their analysis is now clear from our study of the regimes 1 and 8 above. We therefore delegate the calculation of equilibrium demands, supplies and prices of the remaining regimes to the Appendix $\mathrm{B}$ and conclude by listing the defining constraints for all support sets $N_{j}, j=1, \ldots, 8$, in Table 2 .

\begin{tabular}{|l|l|l|l|}
\hline Regime 1 & Regime 2 & Regime 3 & Regime 4 \\
\hline \hline$n_{1} \leq \sigma_{1}$ & $n_{1} \leq \sigma_{1}$ & $n_{1} \leq \sigma_{1}$ & $n_{1} \leq \sigma_{1}$ \\
\hline$n_{2} \leq \sigma_{2} n_{1}+\sigma_{3}$ & $n_{2} \leq \sigma_{2} n_{1}+\sigma_{3}$ & $n_{2} \geq \sigma_{2} n_{1}+\sigma_{3}$ & $n_{2} \geq \sigma_{2} n_{1}+\sigma_{3}$ \\
\hline$n_{3} \leq \sigma_{4} n_{2}+\sigma_{5}$ & $n_{3} \geq \sigma_{4} n_{2}+\sigma_{5}$ & $n_{3} \geq \sigma_{6} n_{2}+\sigma_{7} n_{1}+\sigma_{8}$ & $n_{3} \leq \sigma_{6} n_{2}+\sigma_{7} n_{1}+\sigma_{8}$ \\
\hline
\end{tabular}

\begin{tabular}{|l|l|l|l|}
\hline Regime 5 & Regime 6 & Regime 7 & Regime 8 \\
\hline \hline$n_{1} \geq \sigma_{1}$ & $n_{1} \geq \sigma_{1}$ & $n_{1} \geq \sigma_{1}$ & $n_{1} \geq \sigma_{1}$ \\
\hline$n_{2} \geq \sigma_{9}$ & $n_{2} \leq \sigma_{9}$ & $n_{2} \leq \sigma_{9}$ & $n_{2} \geq \sigma_{9}$ \\
\hline$n_{3} \leq \sigma_{6} n_{2}+\sigma_{10}$ & $n_{3} \leq \sigma_{4} n_{2}+\sigma_{5}$ & $n_{3} \geq \sigma_{4} n_{2}+\sigma_{5}$ & $n_{3} \geq \sigma_{6} n_{2}+\sigma_{10}$ \\
\hline
\end{tabular}

Table 2: The support sets of all equilibrium regimes ${ }^{12}$

The next section turns to the discussion of the relationship between short-run equilibria and the time path of species populations.

\footnotetext{
${ }^{12}$ The parameters $\sigma_{1}, \ldots, \sigma_{10}$ are defined in Appendix B.
} 


\section{The link between short-run ecosystem equilibria and population dynamics}

The information on equilibrium prices, demands and supplies (Appendix B) is now utilized to calculate the equilibrium values of (1) combined with (3) and the parametric specification (5a) - (5c). How this is done is shown in Appendix $\mathrm{C}$ for the polar regimes 1 and 8 . The complete set of differential equations, three for each regime, is listed in Table 3.

The findings summarized in Table 3 distinguish the ecosystem model of the present paper significantly from predator-prey models of the Lotka-Volterra type. The most obvious difference is that in the latter approach the differential equations are defined on the entire domain of populations with parameters that are independent of the values attained by populations. In contrast, the differential equations in Table 3 are valid only on the support sets of the respective equilibrium regimes. Switching regimes means changing the population dynamics. Moreover, Table 3 exhibits the following deviating features of our model:

(i) As in the Lotka-Volterra approach, predator populations impact adversely on the development of prey species but the magnitude of that negative effect depends on the prevailing pattern of scarcity and abundance.

\begin{tabular}{|l|l|l|}
\hline & species 1 & species 2 \\
\hline \hline R1 (000) & $\dot{n}_{1}=\lambda_{1} n_{1}^{2}-\lambda_{2} n_{1}-\lambda_{3} n_{2}+\lambda_{4}$ & $\dot{n}_{2}=\mu_{1} n_{2}^{2}-\mu_{2} n_{2}-\mu_{3} n_{3}+\mu_{4}$ \\
\hline R2 (001) & $\dot{n}_{1}=\lambda_{1} n_{1}^{2}-\lambda_{2} n_{1}-\lambda_{3} n_{2}+\lambda_{5}$ & $\dot{n}_{2}=\mu_{1} n_{2}^{2}-\mu_{5} n_{2}-\mu_{6} n_{3}+\mu_{7}$ \\
\hline R3 (011) & $\dot{n}_{1}=\lambda_{1} n_{1}^{2}-\lambda_{6} n_{1}-\lambda_{7} n_{2}+\lambda_{8}$ & $\begin{array}{c}\dot{n}_{2}=\mu_{8} n_{2}^{2}-\mu_{9} n_{2}+\mu_{10} n_{1}^{2}-\mu_{11} n_{1} \\
+\mu_{12} n_{1} n_{2}-\mu_{6} n_{3}+\mu_{13}\end{array}$ \\
\hline R4 (010) & $\dot{n}_{1}=\lambda_{1} n_{1}^{2}-\lambda_{6} n_{1}-\lambda_{7} n_{2}+\lambda_{8}$ & $\begin{array}{c}\dot{n}_{2}=\mu_{8} n_{2}^{2}-\mu_{14} n_{2}+\mu_{10} n_{1}^{2}-\mu_{15} n_{1} \\
+\mu_{12} n_{1} n_{2}-\mu_{6} n_{3}+\mu_{16}\end{array}$ \\
\hline R5 (110) & $\dot{n}_{1}=-d_{1} n_{1}-\lambda_{7} n_{2}+\lambda_{9}$ & $\dot{n}_{2}=\mu_{8} n_{2}^{2}-\mu_{17} n_{2}-\mu_{3} n_{3}+\mu_{18}$ \\
\hline R6 (100) & $\dot{n}_{1}=-d_{1} n_{1}-\lambda_{3} n_{2}+\lambda_{10}$ & $\dot{n}_{2}=\mu_{1} n_{2}^{2}-\mu_{2} n_{2}-\mu_{3} n_{3}+\mu_{4}$ \\
\hline R7 (101) & $\dot{n}_{1}=-d_{1} n_{1}-\lambda_{3} n_{2}+\lambda_{11}$ & $\dot{n}_{2}=\mu_{1} n_{2}^{2}-\mu_{5} n_{2}-\mu_{6} n_{3}+\mu_{7}$ \\
\hline R8 (111) & $\dot{n}_{1}=-d_{1} n_{1}-\lambda_{7} n_{2}+\lambda_{9}$ & $\dot{n}_{2}=\mu_{8} n_{2}^{2}-\mu_{19} n_{2}-\mu_{6} n_{3}+\mu_{20}$ \\
\hline
\end{tabular}




\begin{tabular}{|l|l|}
\hline & species 3 \\
\hline \hline R1 (000) & $\dot{n}_{3}=\rho_{1} n_{3}^{2}-\rho_{2} n_{3}+\rho_{3}$ \\
\hline R2 (001) & $\dot{n}_{3}=\rho_{4} n_{3}^{2}-\rho_{5} n_{3}+\rho_{6} n_{2}^{2}-\rho_{7} n_{2}+\rho_{8} n_{3} n_{2}+\rho_{9}$ \\
\hline R3 (011) & $\begin{array}{c}\dot{n}_{3}=\rho_{4} n_{3}^{2}-\rho_{10} n_{3}+\rho_{11} n_{2}^{2}-\rho_{12} n_{2}+\rho_{13} n_{1}^{2} \\
\quad-\rho_{14} n_{1}+\rho_{15} n_{3} n_{2}+\rho_{16} n_{3} n_{1}+\rho_{17} n_{2} n_{1}+\rho_{18}\end{array}$ \\
\hline R4 (010) & $\dot{n}_{3}=\rho_{1} n_{3}^{2}-\rho_{2} n_{3}+\rho_{3}$ \\
\hline R5 (110) & $\dot{n}_{3}=\rho_{1} n_{3}^{2}-\rho_{2} n_{3}+\rho_{3}$ \\
\hline R6 (100) & $\dot{n}_{3}=\rho_{1} n_{3}^{2}-\rho_{2} n_{3}+\rho_{3}$ \\
\hline R7 (101) & $\dot{n}_{3}=\rho_{4} n_{3}^{2}-\rho_{5} n_{3}+\rho_{6} n_{2}^{2}-\rho_{7} n_{2}+\rho_{8} n_{3} n_{2}+\rho_{9}$ \\
\hline R8 (111) & $\dot{n}_{3}=\rho_{4} n_{3}^{2}-\rho_{19} n_{3}+\rho_{20} n_{2}^{2}-\rho_{21} n_{2}+\rho_{22} n_{3} n_{2}+\rho_{23}$ \\
\hline
\end{tabular}

Table 3: Population growth of species depending on equilibrium regimes

(ii) The population growth of predator 3 depends on the population of its prey 2 if and only if species 2 is scarce. The population growth of predator 2 depends on the population of its prey, if and only if species 1 is scarce and the resource is abundant. This feature is not characteristic for conventional population models, ${ }^{13}$ since they do not account for scarcity and abundance as determinants of population dynamics.

(iii) The population growth of species 1 depends only on the scarcity or abundance of its prey species, the resource, if and only if species 1 is scarce.

(iv) The observation (ii) does not hold for species 2. As long as its prey species 1 is abundant, the population growth of species 2 depends on its own scarcity or abundance only irrespective of the resource being scarce or abundant.

(v) The population growth of species 3 is not affected by the scarcity or abundance of species 0 and 1, as long as its prey species 2 is abundant. If species 2 is scarce, the scarcity or abundance of the resource is irrelevant for its growth, if and only if species 1 is abundant.

Although the differential equations listed in Table 3 are not too complicated there is no easy way to offer a general solution to the growth or shrinkage of species over time. This is true for the dynamics within one and the same regime and it is also true, a fortiori, for the dynamics when the ecosystem passes through different regimes. In fact, it is interesting and important to know what the sequence of regimes is along the time path of populations, whether the same regime is or can be passed more than once and whether the ecosystem converges, eventually, to a stationary state.

\footnotetext{
${ }^{13}$ Gurney and Nisbet (1998, p. 187) investigate a three-species food chain and discuss similar results. Of course, their food chain is not based on species' optimization.
} 
Table 4 provides some preliminary information on the great variety of viable switches among equilibrium regimes over time. In that table, ' $n_{i} \uparrow \mid R j \rightarrow R k$ ' has the following meaning for $j, k=1, \ldots, 8$ : If $\left(n_{1}, n_{2}, n_{3}\right) \in N_{j}$ and $\dot{n}_{i}>0$ (for $i=1,2$ or 3 ) over successive periods such that $n_{i}$ and only $n_{i}$ eventually hits the boundary of $N_{j}$ then $\left(n_{1}, n_{2}, n_{3}\right)$ enters $N_{k}$, the support set of regime $k$. The first row of Table 4 demonstrates that starting from regime 1 the type of regime switch to occur depends on which population hits the boundary of $N_{1}$ first. The basic message of Table 4 is that many things can happen: Since the population dynamics are shaped by the sequence of regimes passed, the actual time path of all populations crucially depends on the initial values of populations but also on all parameters, since the parameters determine, ceteribus paribus, which population hits the boundary of the prevailing regime first. To obtain more informative results we need to resort to numerical analysis.

\begin{tabular}{|c|c|c|c|c|c|}
\hline$n_{1} \uparrow$ & $\begin{array}{l}\mathrm{R} 1 \rightarrow \mathrm{R} 6 \\
\mathrm{R} 2 \rightarrow \mathrm{R} 7 \\
\mathrm{R} 3 \rightarrow \mathrm{R} 8 \\
\mathrm{R} 4 \rightarrow \mathrm{R} 5\end{array}$ & $n_{2} \uparrow$ & $\begin{array}{l}\mathrm{R} 1 \rightarrow \mathrm{R} 4 \\
\mathrm{R} 2 \rightarrow \mathrm{R} 3 \\
\mathrm{R} 6 \rightarrow \mathrm{R} 5 \\
\mathrm{R} 7 \rightarrow \mathrm{R} 8\end{array}$ & $n_{3} \uparrow$ & $\begin{array}{l}\mathrm{R} 1 \rightarrow \mathrm{R} 2 \\
\mathrm{R} 4 \rightarrow \mathrm{R} 3 \\
\mathrm{R} 5 \rightarrow \mathrm{R} 8 \\
\mathrm{R} 6 \rightarrow \mathrm{R} 7\end{array}$ \\
\hline
\end{tabular}

Table 4: Regime switches during population growth

\section{Numerical analysis of population growth}

In this section we provide six numerical examples. It should be pointed out that the parameters chosen are not based on empirical estimates. Our aim is rather to clarify the potential of our equilibrium approach as compared to the well-established models of the Verhulst-Pearl type (growth of a single species) and of the Lotka-Volterra type (growth in a predator-prey context). For reasons of comparison with the aforementioned models as well as for simplicity of exposition we introduce a partial equilibrium component into our subsequent calculations by keeping two populations (examples 1-3), one population (examples 4 and 5) constant or exogenously decreasing one population period by period (example 6). This is clearly a rather severe restriction. But note that keeping two populations or one population constant does not amount to consider an ecosystem with a single species or with two species, respectively. In case where only the population of species 1 is endogenous, species 2 and 3 are still present and active in terms of preying and being preyed. In fact, with $\dot{n}_{i}$ from (1), $\dot{n}_{i} \neq 0$ is not excluded for $i=2,3$. Keeping $n_{2}$ and $n_{3}$ constant rather means that for $i=2,3$ any endogenous population change is immediately and fully compensated by an opposite flow of members of species $i$ into or out of 
the ecosystem under consideration. These compensating flows may be interpreted either as migration of species $i$ from or into neighboring ecosystems or as the result of a special harvesting or wildlife care policy of humans designed to keep the populations of species 2 and 3 constant.

Table 5 presents an overview of the examples to be discussed below. The numerical specification of parameters on which these examples are based is given in Appendix C. The examples 1 - 3 are built on the same set of parameter values while the parameter values are reset ${ }^{14}$ for each of the examples $4-6$.

\begin{tabular}{|c|c|c|c|c|}
\hline $\begin{array}{c}\text { Example } \\
\text { no. }\end{array}$ & $\begin{array}{c}\text { variable } \\
\text { population(s) }\end{array}$ & $\begin{array}{c}\text { initial } \\
\text { regime }\end{array}$ & $\begin{array}{c}\text { intermediate } \\
\text { regime }\end{array}$ & $\begin{array}{c}\text { final regime } \\
\text { and steady state }\end{array}$ \\
\hline \hline 1 & $n_{1}$ & $\mathrm{R} 2(001)$ & - & $\mathrm{R} 7(101)$ \\
\hline 2 & $n_{1}$ & $\mathrm{R} 4(010)$ & $\mathrm{R} 1(000)$ & $\mathrm{R} 6(100)$ \\
\hline 3 & $n_{3}$ & $\mathrm{R} 1(000)$ & - & $\mathrm{R} 1(000)$ \\
\hline 4 & $n_{2}, n_{3}$ & $\mathrm{R} 7(101)$ & - & $\mathrm{R} 8(111)$ \\
\hline 5 & $n_{1}, n_{2}$ & $\mathrm{R} 2(001)$ & - & $\mathrm{R} 7(101)$ \\
\hline 6 & $n_{1}, n_{3}$ & $\mathrm{R} 8(111)$ & - & $\mathrm{R} 7(101)$ \\
\hline
\end{tabular}

Table 5: Variable populations, regimes and regime switches in examples 1 to 6

We start with fixing two populations which allows us to compare our model with the Verhulst-Pearl framework of analysis. Before we carry out the numerical calculations of the pertinent examples 1 - 4 it is worth pointing out that our model gives rise to a parametric (rather than numerical) solution to this scenario. To see this observe from Table 3 that the differential equations characterizing the growth of species $i$ is either of type

$$
\dot{n}_{i}=\gamma n_{i}^{2}-\delta n_{i}+\varepsilon
$$

or of type

$$
\dot{n}_{i}=-\eta n_{i}+\theta
$$

if all populations $j, j \neq i$, are kept constant $(i, j=1,2,3)$. The differential equations (14)

\footnotetext{
${ }^{14}$ Obviously, it is through the appropriate choice of parameter values (and through fixing the initial values of populations) that the inequalities in Table 2 and hence the initial equilibrium regime are selected.
} 
and (15) can be explicitly solved. Separating variables we obtain ${ }^{15}$

$$
\int \mathrm{d} t=\int \frac{\mathrm{d} n_{i}}{\gamma n_{i}^{2}-\delta n_{i}+\varepsilon}= \begin{cases}\frac{2}{\sqrt{-D}} \arctan \left(\frac{2 \gamma n_{i}-\delta}{\sqrt{-D}}\right) & \text { for } D<0 \\ -\frac{2}{2 \gamma n_{i}-\delta} & \text { for } D=0 \\ \frac{2}{\sqrt{D}} \operatorname{artanh}\left(\frac{2 \gamma n_{i}-\delta}{\sqrt{D}}\right) & \text { for } D>0\end{cases}
$$

where $D:=\delta^{2}-4 \gamma \varepsilon$, and

$$
\int \mathrm{d} t=\int \frac{\mathrm{d} n_{i}}{-\eta n_{i}+\theta}=-\frac{1}{\eta} \ln \left|-\eta n_{i}+\theta\right| .
$$

We rearrange (16) and (17) and account for $n_{i}(0)=\kappa$ to establish

$$
n_{i}(t)= \begin{cases}\frac{\sqrt{-D} \tan \left[\frac{1}{2} t \sqrt{-D}+\arctan \left(\frac{2 \gamma \kappa-\delta}{\sqrt{-D}}\right)\right]+\delta}{2 \gamma} & \text { for } D<0, \\ \frac{\delta}{2 \gamma}+\frac{2}{\gamma(2 \gamma \kappa-\delta)}-\frac{t}{\gamma} & \text { for } D=0, \\ \frac{\sqrt{D} \tanh \left[\frac{1}{2} t \sqrt{D}+\operatorname{artanh}\left(\frac{2 \gamma \kappa-\delta}{\sqrt{D}}\right)\right]+\delta}{2 \gamma} & \text { for } D>0,\end{cases}
$$

and

$$
n_{i}(t)=\left(\kappa-\frac{\theta}{\eta}\right) \exp ^{-\eta t}+\frac{\theta}{\eta}
$$

Now we turn to the numerical simulations which are conducted with help of the computer program Mathematica. ${ }^{16}$

Example 1: In regime 2 the resource and the biomass of species 1 are abundant, $p_{0}=0, p_{1}=0$, and the price of biomass of species 2 is positive, $p_{2}>0$. Inserting the parameter values (listed in the Appendix C) into the differential equation presented in Table 3 (column "species 1", row "R2(001)") and assuming $\bar{n}_{2}=30$ we obtain

$$
\dot{n}_{1}=\frac{1}{5} n_{1}^{2}-4 n_{1}+\frac{24}{5} .
$$

The explicit solution of the differential equation (20) is given by the tanh-function in the third row of (18). According to Table $2,\left(n_{1}, \bar{n}_{2}, \bar{n}_{3}\right) \in N_{2}$ requires $n_{1} \leq \sigma_{1}$ with $\sigma_{1}=2510$. In the first short-run period we set the population of species 1 equal to $n_{1}(0)=20$. The left panel of Figure 1 shows that during the first time interval $[0,0.6]$ the population of species 1 grows slowly but after $t=0.6$ we observe an approximately exponential and rapid growth. From $t=0$ to $t=0.77$ the population of species 1 has increased from 20 to

\footnotetext{
${ }^{15}$ For the solution of the second integral in (16) compare Sydsæter, Strøm and Berck (1998).

${ }^{16}$ The program for simulations is available from the authors upon request.
} 
$n_{1}=\sigma_{1}=2510$. Since $\left(\sigma_{1}, \bar{n}_{2}, \bar{n}_{3}\right)$ is a boundary point of $N_{2}$ and since $\dot{n}_{1}>0$ at $t=0.77$ the resource is no longer abundant, its price $p_{0}$ switches from zero to positive, implying that the population vector $\left(n_{1}, \bar{n}_{2}, \bar{n}_{3}\right)$ leaves $N_{2}$ and enters $N_{7}$ as defined in Table 2 . Now we use the same parameter values (Appendix C) to determine the population growth of species 1 in regime 7 as

$$
\dot{n}_{1}=-2 n_{1}+\frac{6275263}{5}
$$

for $n_{1} \geq 2510$. The right panel of Figure 1 illustrates the continuing growth of the population of species 1 in regime 7 and it shows the growth levelling off such that species 1 approaches its steady state level $n_{1}=627526.26$.
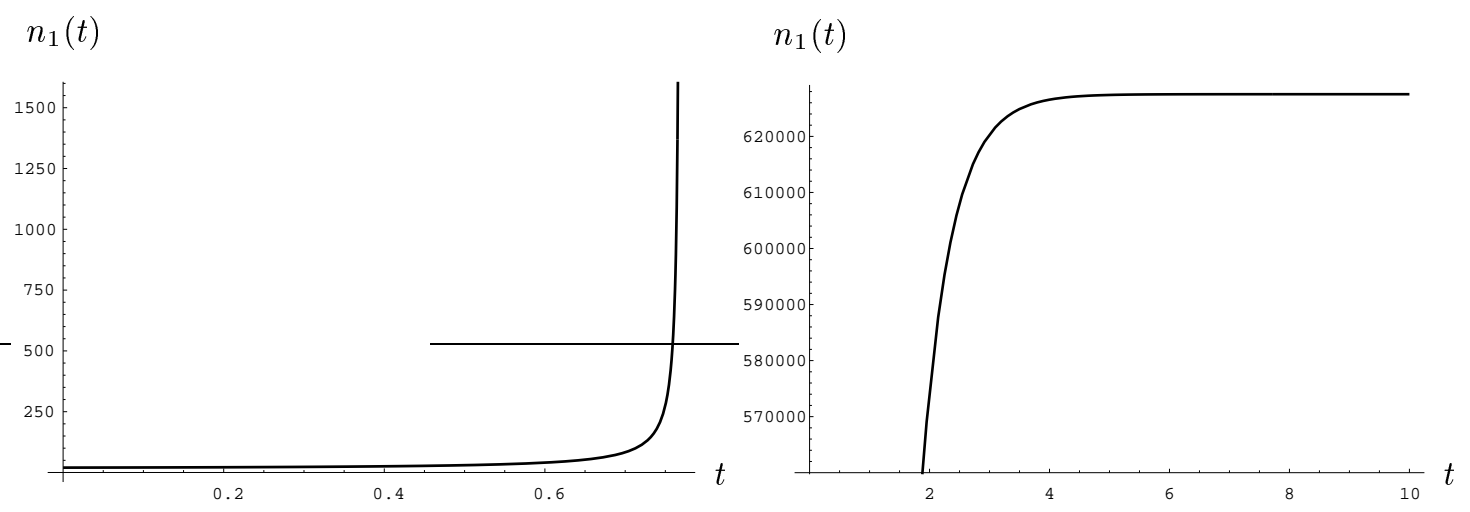

Figure 1: Population growth of species 1 in example 1 (regimes 2 and 7)

Example 2: The differential equations describing population growth in regime 4 (010), regime 1 (000) and regime 6 (100) are, respectively,

$$
\begin{aligned}
& \dot{n}_{1}=\frac{n_{1}^{2}}{5}-\frac{13}{3} n_{1}-\frac{116}{3} \\
& \dot{n}_{1}=\frac{n_{1}^{2}}{5}-4 n_{1}-\frac{215}{5} \\
& \dot{n}_{1}=\frac{6274761}{5}-2 n_{1} .
\end{aligned}
$$

The solutions to (22a) and (22b) involve the tanh-function and the solution to (22c) is of type (19). We start in regime 4 (010) where the resource and biomass of species 2 are abundant whereas the biomass of species 1 is scarce. The population of species 2 is kept constant at the level $\bar{n}_{2}=600$ and the population of species 1 is $n_{1}(t)=30$ at $t=0$. According to Table 2 the relevant constraints for regime 4 are:

$$
\begin{aligned}
n_{1} & \leq \sigma_{1}=2510 \\
n_{2}=600 & \geq \sigma_{2} n_{1}+\sigma_{3}=25 n_{1}-\frac{445}{2} .
\end{aligned}
$$




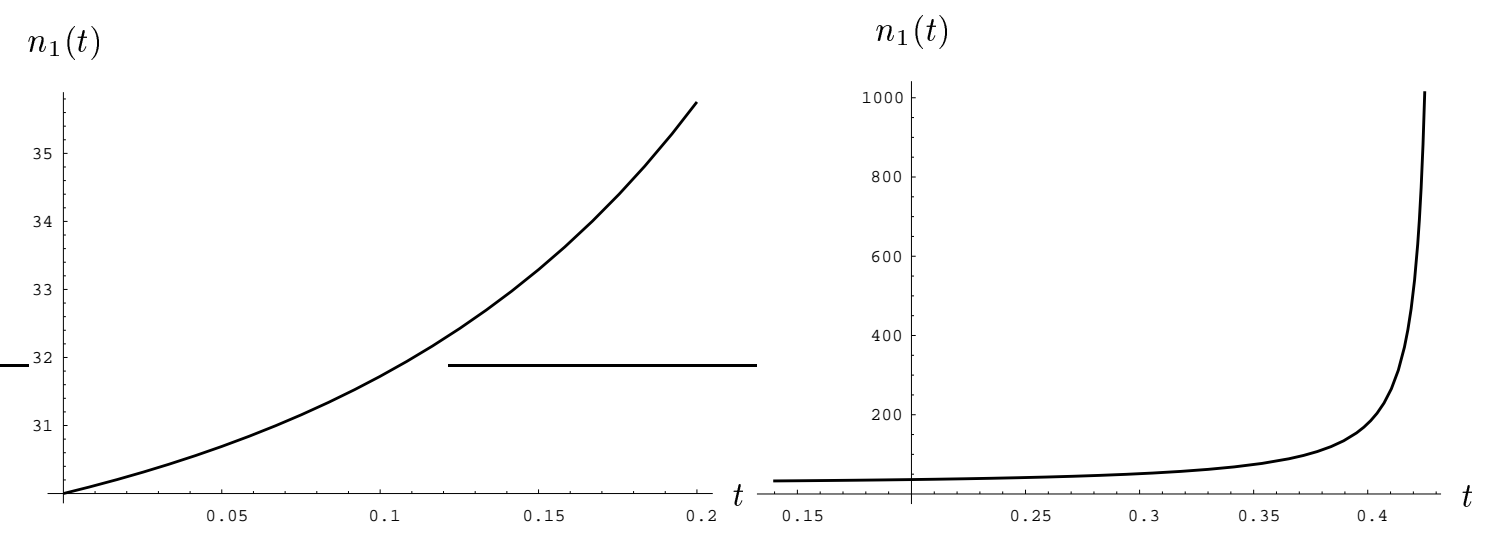

Figure 2: Population growth of species 1 in example 2 (regimes 4 and 1)

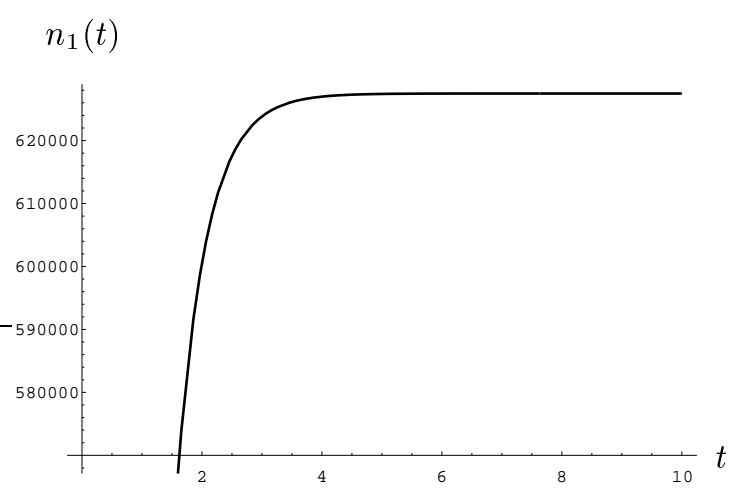

Figure 3: Population growth of species 1 in example 2 (regime 6)

For $t=0$, the population vector $\left(n_{1}, \bar{n}_{2}, \bar{n}_{3}\right)$ satisfies (23a) and (23b) since $30<2510$ and $600>527.5$. The left panel of Figure 2 shows that the population of species 1 grows from the beginning. Therefore, $n_{1}$ is bound to hit the boundary (23b) implying that the biomass of species 1 is no longer scarce. When the price $p_{1}$ declines to zero at $t=0.14$ with $n_{1}(0.14)=32.9$ the ecosystem switches from regime 4 to regime 1 . In regime 1 all prices are zero and species are in a land of utter abundancy. The population of species 1 keeps growing as shown in the right panel of Figure 2 until the resource gets scarce at $t=0.42$. In regime 1 the population has increased from $n_{1}(0.14)=32.9$ to $n_{1}(0.42)=\sigma_{1}=2510$. At the point in time $t=0.42$ the ecosystem leaves regime 1 and enters regime 6 where the population of species 1 grows at decreasing rates until it converges, eventually, to its steady state level 62575.5 as illustrated in Figure 3.

Conceptually the left and right panel of Figure 1 are segments of a single diagram and the same is true for the three diagrams contained in Figures 2 and $3 .{ }^{17}$ But even

\footnotetext{
${ }^{17}$ The only reason for presenting the segments separately is that integrated diagrams are less informative
} 
without putting all segments together it is easy to see that our growth curve has the shape of a logistic growth curve quite similar to the curve implied by the generalized VerhulstPearl equation (Verhulst 1838, Pearl 1930, Rosen 1984). There are two main differences between our result and the 'traditional' logistic growth curve. First, the latter follows from a single differential equation. In contrast, our logistic growth curve is implied by more than one differential equation depending on the number of regimes passed. In example 1 the differential equation is made up of two segments and in example 2 it is made up of three segments. The second and more important difference is that our logistic growth curve is deducted from optimizing species' transactions in the ecosystem and these transactions in turn determine whether resources and biomass of species are abundant or scarce. In contrast, the Verhulst-Pearl equation ignores the impact of scarcity and abundance of resources and biomass on the population dynamics.

In the next example we demonstrate that another time path of population growth of a single species is possible.

Example 3: The populations of species 1 and 2 are assumed to be $\bar{n}_{1}=100$ and $\bar{n}_{2}=50$ so that the population growth of species 3 is given by

$$
\dot{n}_{3}=\frac{2}{15} n_{3}^{2}-\frac{8}{3} n_{3}+\frac{5}{6} .
$$

Starting with $n_{3}(0)=15$ the left panel of Figure 4 illustrates the decrease in the population $n_{3}$. Obviously, the prey population (species 2) is too small to sustain the population of species 3. Confirming our expectation, the population of species 3 does not go extinct but converges to a steady state as shown in the right panel of Figure 4.

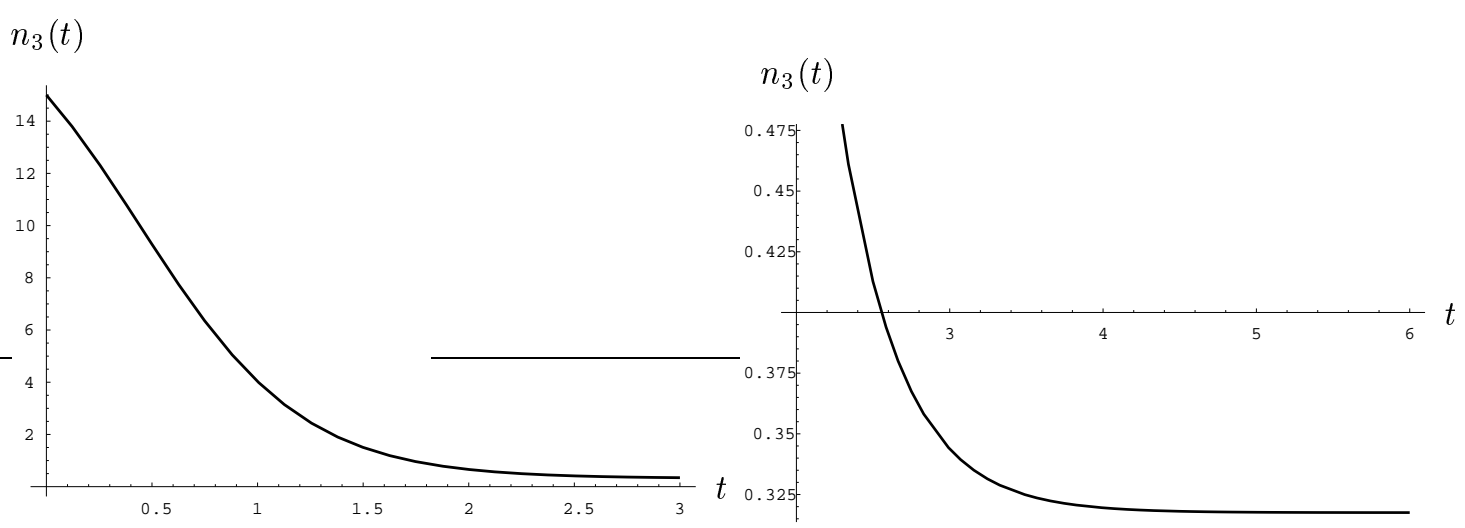

Figure 4: Population growth of species 3 in example 3

Example 4: In this example we turn to the population growth of two species. The ecosystem begins in regime 7 (101), the population of species 1 is assumed to be $\bar{n}_{1}=10$ due to the need of choosing uniform scales. 
and the growth of species 2 and 3 is given by

$$
\begin{aligned}
& \dot{n}_{2}=\frac{1}{2} n_{2}^{2}-2.91 n_{2}-0.91 n_{3}+21.81 \\
& \dot{n}_{3}=0.04 n_{3}^{2}+0.08 n_{3} n_{2}-1.99 n_{3}+0.04 n_{2}^{2}-0.99 n_{2}+5.95
\end{aligned}
$$

using the parameter values listed in the Appendix C. The population of species 2 turns out to increase more rapidly than the population of species 3 , hits its boundary line $\sigma_{9}=$ 2.5 , and subsequently the populations of both species grow according to the differential equations of regime 8 (111),

$$
\begin{aligned}
& \dot{n}_{2}=-n_{2}-0.91 n_{3}+9.26 \\
& \dot{n}_{3}=0.04 n_{3}^{2}-1.86 n_{3}+3.73
\end{aligned}
$$

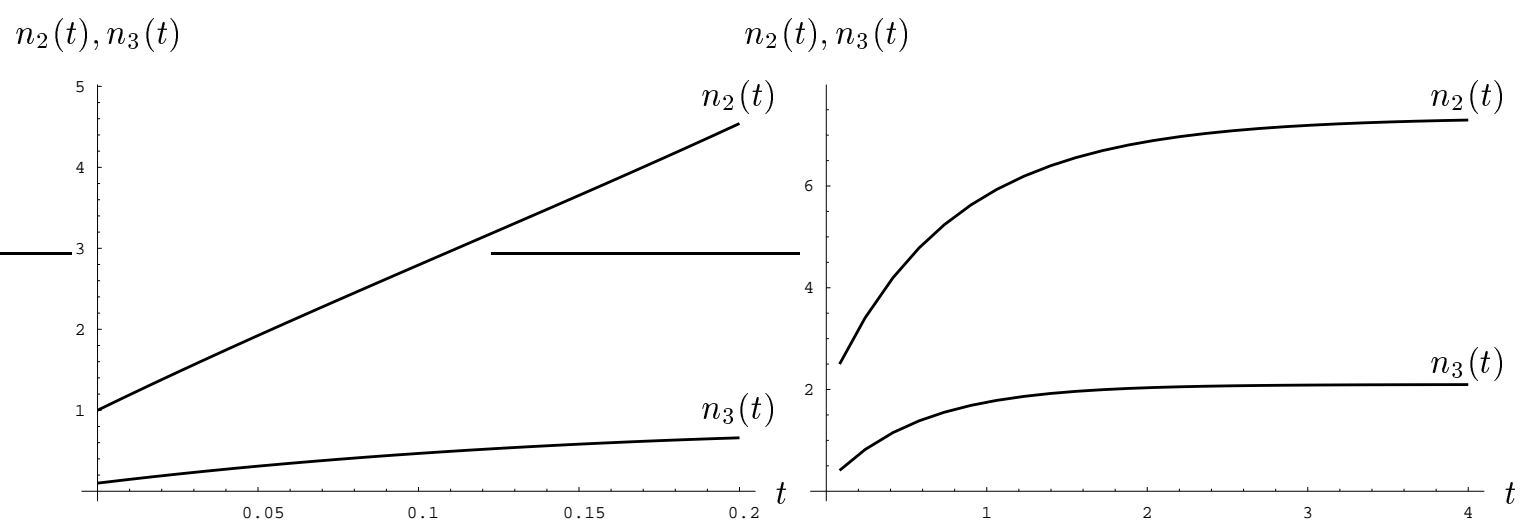

Figure 5: Population growth of species 2 and 3 in example 4 (regimes 7 and 8)

Population growth in regime 7 is illustrated in the left panel of Figure 5, and growth in regime 8 in the right panel of Figure 5. Lumped together, the left and right panels of Figure 5 exhibit logistic growth curve for the population of species 2 (even though the strict convexity of the curve in the left panel of Figure 5 is hardly visible), and they displays ever decreasing and eventually fading growth of the population of species 3 . Thus example 4 demonstrates that coexistence of species is a feasible outcome of our model. Note that in steady state regime 8 all biomasses and resources are scarce.

Figure 6 shows the trajectories in regimes 7 and 8 which elucidate that the prey population (species 2) grows more rapidly than the predator population (species 3).

Example 5: This example is designed to demonstrate that coexistence is also feasible in ecosystems where at least one species is abundant. Making use of parameter values given 


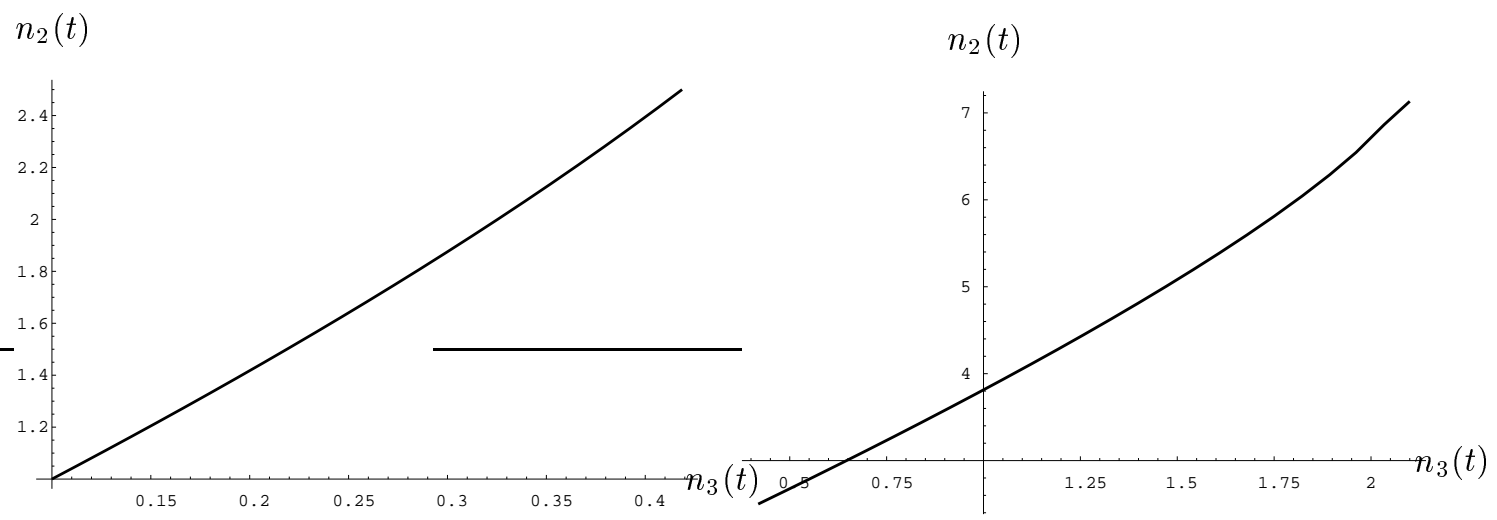

Figure 6: Trajectories in example 4 (regimes 7 and 8)

in the Appendix $\mathrm{C}$, the population growth is described by

$$
\begin{gathered}
\dot{n}_{1}=\frac{9}{20} n_{1}^{2}-25 n_{1}-3 n_{2}+155, \\
\dot{n}_{2}=9 n_{2}^{2}-250.15 n_{2}+904.48
\end{gathered}
$$

in the initial regime 2. As shown in the left panels of Figures 7 and 8 both populations grow but the population of species 1 grows faster and therefore $n_{1}$ hits its boundary line $\sigma_{1}=350.17$ first. After the switch to regime 7 the population growth of species 2 is still given by $(27 \mathrm{~b})$ but that of species 1 is now

$$
\dot{n}_{1}=-10 n_{1}-3 n_{2}+50080 .
$$

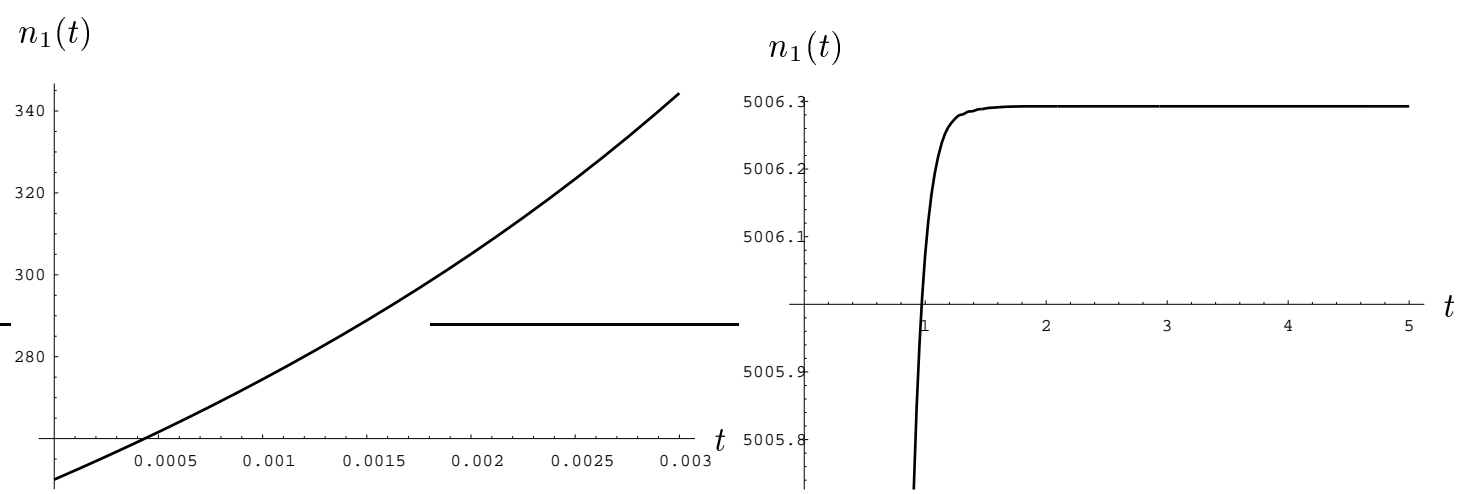

Figure 7: Population growth of species 1 in example 5 (regimes 2 and 7)

The right panels of Figures 7 and 8 display growth in regime 7 where both populations eventually reach a steady state and Figure 9 provides the information that species 1 grows 

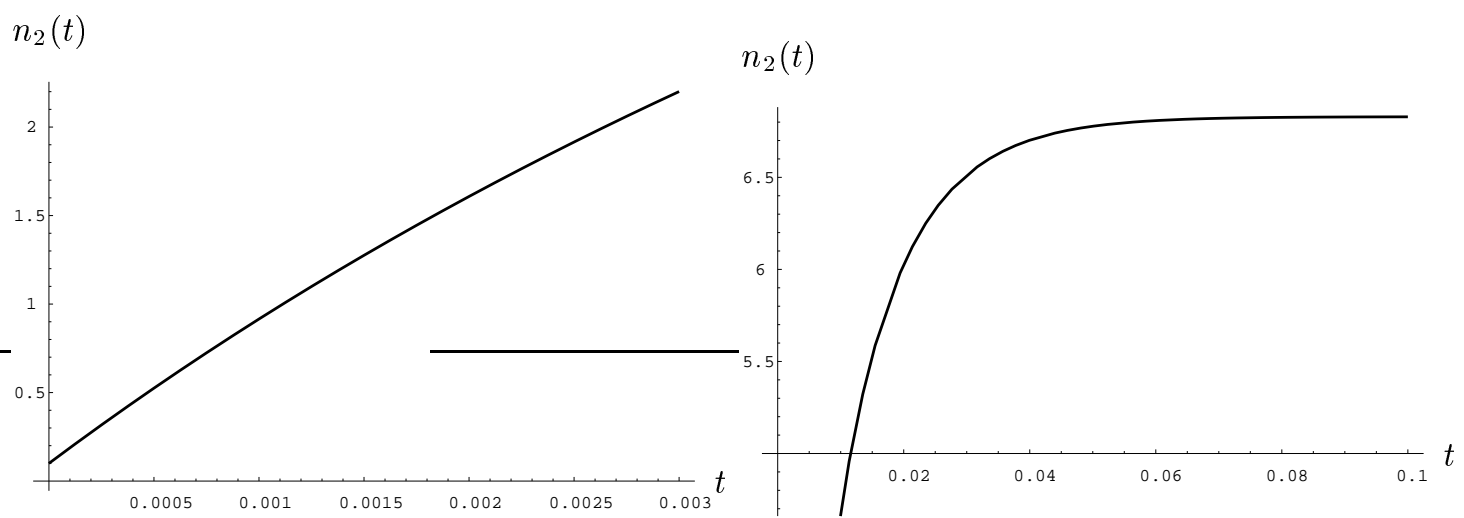

Figure 8: Population growth of species 2 in example 5 (regimes 2 and 7 )

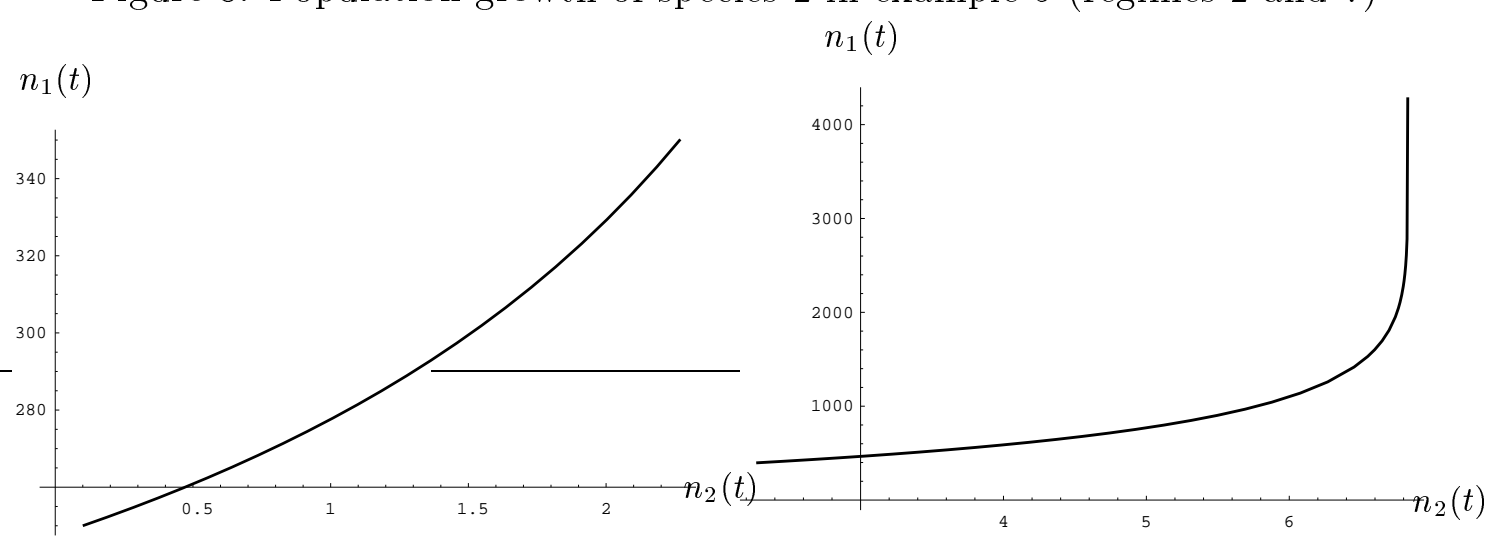

Figure 9: Trajectories in example 5 (regimes 2 and 7)

more rapidly than species 2 . It is interesting to observe that the examples 4 and 5 exhibit a common feature: The population growth of the prey species is logistic while that of the predator species is positive but strictly declining. ${ }^{18}$

Example 6: In our last example we study the growth of species 1 and 3 when species 2 is exogenously decreased period by period, say through harvesting. The population of species 2 is assumed to take the functional form

$$
n_{2}(t)=100-500 t
$$

The ecosystem starts in regime 8 (111) where all biomasses are scarce. The population growth of species 1 and 3 is given by

$$
\begin{aligned}
& \dot{n}_{1}=-\frac{n_{1}}{2}-\frac{n_{2}}{6}+204.17 \\
& \dot{n}_{3}=\frac{n_{3}^{2}}{242}+0.0001 n_{3} n_{2}-9.86 n_{3}+1.15 \cdot 10^{-6} n_{2}^{2}+0.0002 n_{2}+0.01,
\end{aligned}
$$

\footnotetext{
${ }^{18}$ Examples 4 and 5 do not generate 'traditional' predator-prey cycles. To obtain these cycles one has to choose a parameter constellation such that in one regime the prey population increases while the predator population decreases, and such that in another regime the predator population increases while the prey population decreases. Switching between those regimes would yield cycles. Our conjecture is that these parameter constellation exist but generating predator-prey cycles is beyond the scope of the paper.
} 
and we set $n_{1}(0)=300$ and $n_{2}(0)=150$.
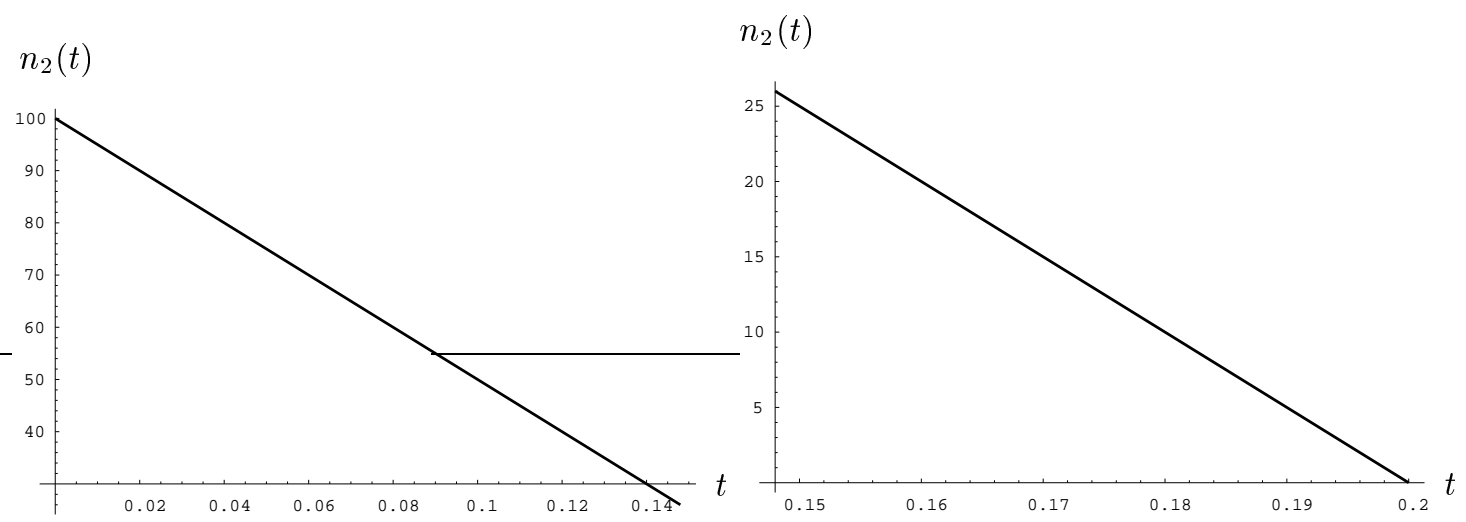

Figure 10: Population growth of species 2 in example 6 (regimes 8 and 7)

Exogenously reducing the population of species 2 which is shown in the left panel of Figure 10 has two consequences: (i) the amount of predators species 1 faces decreases such that the population of species 1 increases (see left panel of Figure 11); (ii) the amount of prey being available to species 3 decreases such that the population of species 3 decreases (see left panel of Figure 13.) The continuously reduction of species 2 implies that species 2 hits the boundary point $n_{2}=\sigma_{9}=25.01$ at $t=37 / 250$ and the regime switches from R8 to R7 (101) implying that the biomass of species 1 becomes abundant. In regime 7 the population growth is then characterized by

$$
\begin{aligned}
& \dot{n}_{1}=-\frac{n_{1}}{2}-n_{2}+225.01 \\
& \dot{n}_{3}=\frac{n_{3}^{2}}{242}+0.0008 n_{3} n_{2}-10 n_{3}+0.00004 n_{2}^{2}-0.0004 n_{2} .
\end{aligned}
$$

The effects we again observe are (i) and (ii) which are illustrated in the right panels of Figures 11 and 13. At $t=0.2$ the population of species 2 goes extinct such that $n_{2}(t)=0$ for all $t \geq 0.2$ and (31a) and (31b) simplify to

$$
\begin{aligned}
& \dot{n}_{1}=-\frac{n_{1}}{2}+225.01, \\
& \dot{n}_{3}=\frac{n_{3}^{2}}{242}-10 n_{3} .
\end{aligned}
$$



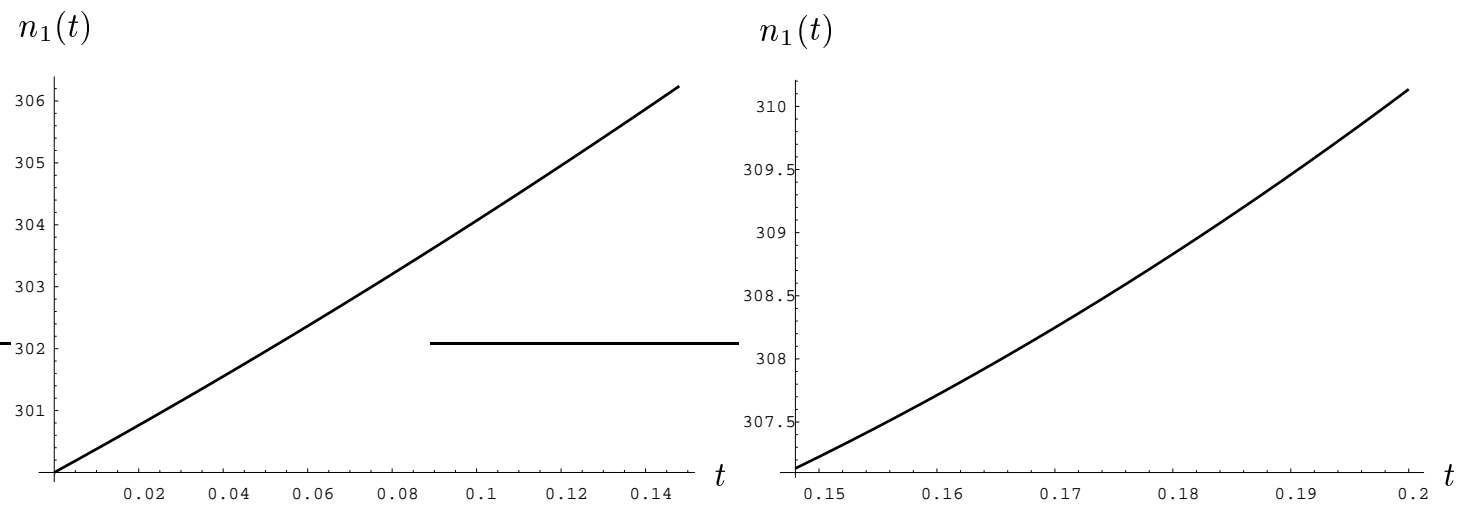

Figure 11: Population growth of species 1 in example 6 (regimes 8 and 7 )

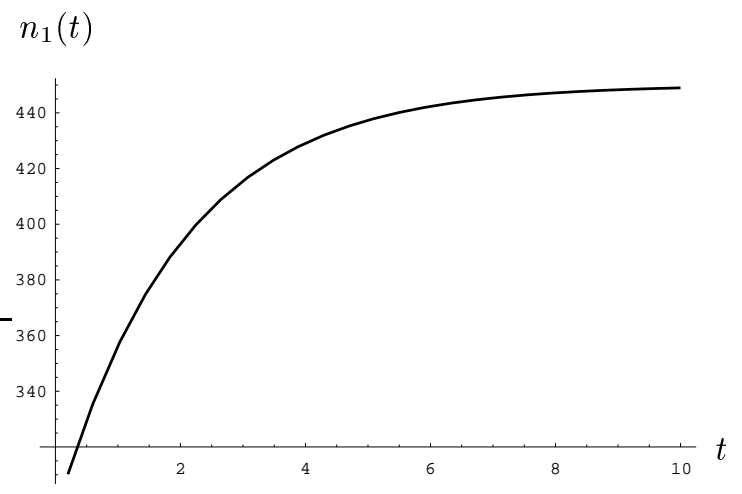

Figure 12: Population growth of species 1 in example 6 (regime 7 and $n_{2}=0$ )
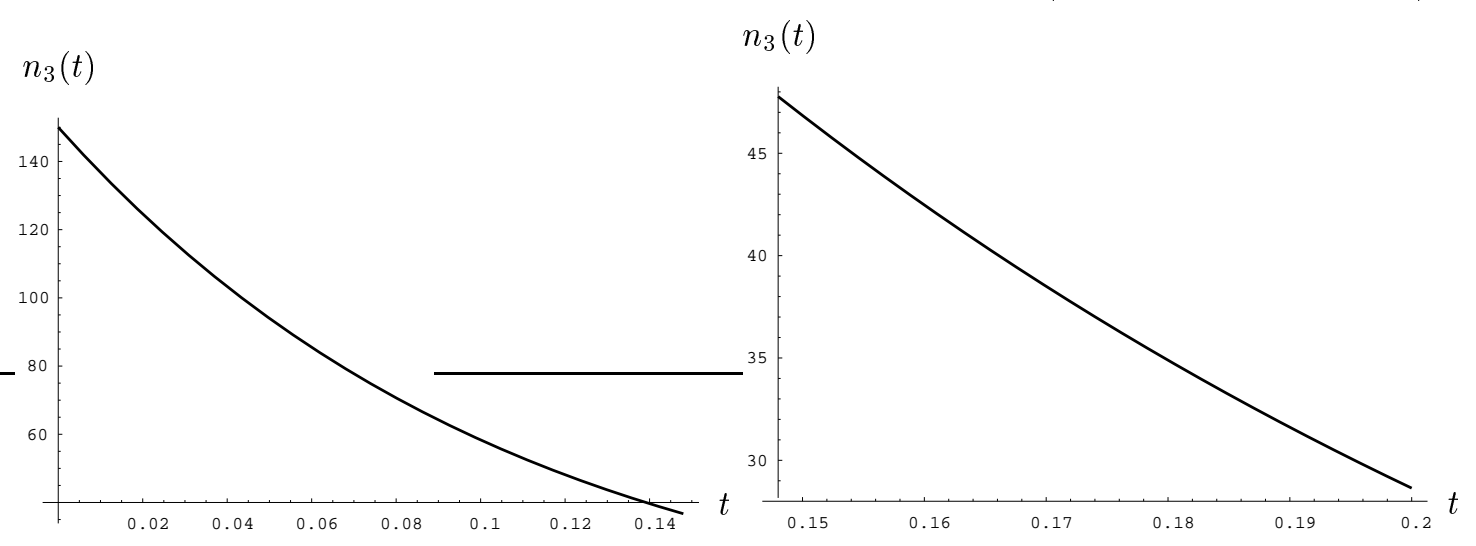

Figure 13: Population growth of species 3 in example 6 (regimes 8 and 7)

Confirming our expectation, the population of species 3 goes extinct (see Figure 14), too, while the population of species 1 runs into its steady state level 448.98 (compare Figure $12)$. 


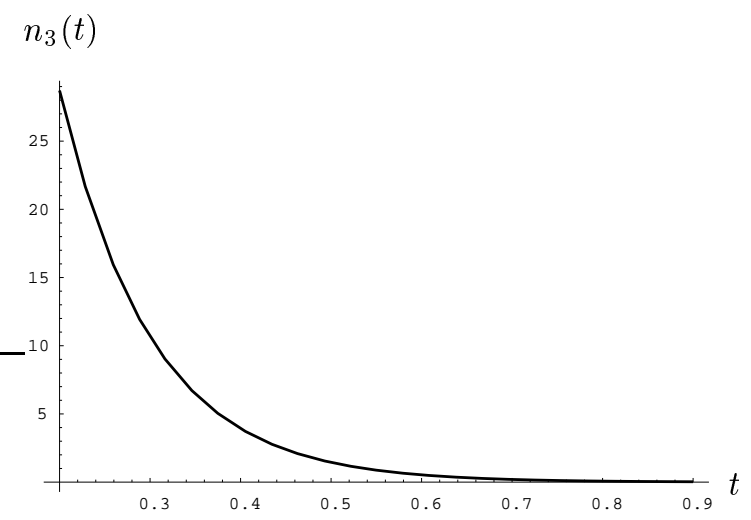

Figure 14: Population growth of species 3 in example 6 (regime 7 and $n_{2}=0$ )

\section{Concluding remarks}

This paper derives population dynamics in an ecosystem consisting of a three species food chain with explicitly modeled predator prey interaction. Unlike the bulk of the literature that takes differential population equations as the basic building blocks of modeling ecosystem interaction we aim at a theoretical 'foundation' of population growth. To that end, we assume that species behave as if they maximize biomass intake and introduce prices as indicators of scarcity and abundance ${ }^{19}$ of species' biomass. Moreover, we make a distinction between the short run where the biomass transactions take place and the long run where populations grow or shrink. This methodology is quite familiar to economists who assume, e.g., the capital stock being constant in the short-run period while they are interested in the time path of capital formation in the long run. The decisive link between short-run equilibrium allocations and population growth established in our ecosystem model yields a set of differential equations governing the population dynamics. In principle, we obtain differential equations which are comparable to those suggested by Lotka and Volterra. However, while in models of Lotka-Volterra type a single differential equation is introduced (in an ad hoc way), one for each species, we deduce from more basic assumptions a set of differential equations for each population depending on the pattern of scarcity and abundance in biomass 'markets'.

The numerical analysis of Section 5 is meant to be a methodological contribution without claiming empirical relevance of the parameters chosen. It does not fully exploit the rich implications of the ecosystem model presented here, since we made use of the ceteribus paribus clause regarding population changes. Studying the full-scale dynamics is beyond the scope of the present paper, and therefore we refrain from drawing generalizing

\footnotetext{
${ }^{19}$ The allowance for abundance is a main difference between prior contributions taking the route we have chosen, especially the works of Tschirhart (2000, 2002).
} 
conclusions from the examples presented in Section 5. Among the challenging questions that remain (almost) unanswered here are the following: How does an ecosystem develop and which regimes does it pass starting from a scenario of utter abundance? Is there a tendency (or even an 'ecological law') of diminishing abundance such that each ecosystem eventually ends up in a state of total scarcity ${ }^{20}$ (regime 8)? Under which conditions can we expect stationary states (that are locally or globally stable)? What are the preconditions for coexistence, persistence and extinction?

It has been stressed that the primary focus of the present paper is on methodology and conceptual analysis. But empirical applications in case studies ought to be the ultimate end which is admittedly in great distance but not entirely unrealistic. Tschirhart (2000, 2002) points to various possibilities of tapping available relevant data to find realistic parameter values and to test various hypotheses implied by the formal model.

\section{References}

Berezovskaya F., Karev G. and R. Ariditi (2001), Parametric analysis of the ratio-dependent predator-prey model, Journal of Mathematical Biology 43, 221-246.

Cantrell R. S. and C. Cosner (2001), On the dynamics of predator-prey models with the Beddington-DeAngelis functional response, Journal of Mathematical Analysis and Applications 257, 206-222.

Crocker T. D. and J. Tschirhart (1992), Ecosystems, externalities and economies, Environmental and Resource Economics 2, 551-567.

Gurney W. S. C. and R. M. Nisbet (1998), Ecological dynamics, Oxford University Press, New York.

Hannon B. (1976), Marginal product pricing in the ecosystem, Journal of Theoretical Biology 56, 253-267.

Houston A. and J. McNamara (1999), Models of adaptive behaviour, Cambridge University Press, Cambridge.

Lima S. L. and L. M. Dik (1990), Behavioral decisions made under the risk of predation: a review and prospectus, Canadian Journal of Zoology 68, 619-640.

\footnotetext{
${ }^{20}$ As shown in Table 5 regime 8 is only one of a number of steady state regimes. But it is an open question whether this feature carries over in scenarios where all populations are endogenous. Moreover, abundance raises the issue of immigrating species filling 'ecological niches'.
} 
Lotka A. J. (1925), Elements of physical biology, Williams und Wilkins, Baltimore.

Pearl R. (1930), The biology of population growth, Knopf, New York.

Pethig R. and J. Tschirhart (2001), Microfoundations of population dynamics, Journal of Bioeconomics 3, 27-49.

Pimm S. (1982), Food webs, Chapman and Hall, Cambridge.

Rosen G. (1984), Characterizing conditions for generalized Verhulst logistic growth of a biological population, Bulletin of Mathematical Biology 46, 963-965.

Stephens D. W. and J. R. Krebs (1986), Foraging theory, Princeton University Press, Princeton.

Sydsæter K., Strøm A. and P. Berck (1998) Economists' mathematical manual, Springer, Berlin et al.

Tschirhart J. (2000), General equilibrium of an ecosystem, Journal of Theoretical Biology 203, 13-32.

Tschirhart J. (2002), Resource competition among plants: from optimizing to community structure, Ecological Modelling 148, 191-212.

Verhulst P. F. (1838), Notice sur la loi que la population suit dans son accroissement, Correspondance Mathematique et Physique 10, 113-121.

Volterra V. (1926), Variazionie fluttuazioni del numero d'individui in specie animali conviventi, Mem. Acad. Lincei. 2, 31-113 (Variations and Fluctuations of a Number of Individuals in Animal Species Living Together, Translation In Chapman R. N. (1931), Animal Ecology, 409-448, Mc Graw Hill, New York. 


\section{Appendix}

\section{Appendix A: Proof of Propositions 1 and 2}

First, we need to introduce some notation. Starting from (5a), let

$$
F^{i}\left(s_{i}, x_{i-1}, \bar{x}_{i}\right):=B^{i}\left[H^{i}\left(x_{i-1}\right), s_{i}, x_{i-1}\right]=\left(a_{i} n_{i}-e_{i}-f_{i} p_{i-1}\right) x_{i-1}-\frac{c_{i}}{2} x_{i-1}^{2}-s_{i}
$$

where $s_{i}=\min \left[\bar{x}_{i}, r_{i}\right]$ and $r_{i}=g_{i} p_{i}+k_{i} x_{i-1}$. Define also $^{21}$

$$
\begin{aligned}
F^{i}\left(s_{i} \equiv r_{i}, x_{i-1}\right) & :=\left(a_{i} n_{i}-e_{i}-k_{i}-f_{i} p_{i-1}\right) x_{i-1}-\frac{c_{i}}{2} x_{i-1}^{2}-g_{i} p_{i}, \\
F^{i}\left(s_{i} \equiv \bar{x}_{i}, x_{i-1}\right) & :=\left(a_{i} n_{i}-e_{i}-f_{i} p_{i-1}\right) x_{i-1}-\frac{c_{i}}{2} x_{i-1}^{2}-\bar{x}_{i}, \\
\hat{x}_{i-1} & :=\arg \max F^{i}\left(s_{i} \equiv g_{i} p_{i}+k_{i} x_{i-1}, x_{i-1}\right)=\frac{a_{i} n_{i}-e_{i}-k_{i}-f_{i} p_{i-1}}{c_{i}}, \\
\hat{r}_{i} & :=g_{i} p_{i}+k_{i} \hat{x}_{i-1} \\
\tilde{x}_{i-1} & :=\arg \max F^{i}\left(s_{i} \equiv \bar{x}_{i}, x_{i-1}\right)=\frac{a_{i} n_{i}-e_{i}-f_{i} p_{i-1}}{c_{i}} \\
\left(s_{i}^{*}, x_{i-1}^{*}\right) & :=\arg \max F^{i}\left(s_{i}, x_{i-1}, \bar{x}_{i}\right) .
\end{aligned}
$$

From these definitions follows immediately

$$
F^{i}\left(s_{i}^{*}, x_{i-1}^{*}, \bar{x}_{i}\right)=\max \left[F^{i}\left(s_{i} \equiv \bar{x}_{i}, \tilde{x}_{i-1}\right), F^{i}\left(s_{i} \equiv \hat{r}_{i}, \hat{x}_{i-1}\right)\right]
$$

which is equivalent to

$$
\begin{array}{r}
\text { A1: } \quad\left(s_{i}^{*}, x_{i-1}^{*}\right)=\left\{\begin{array}{c}
\left(\bar{x}_{i}, \tilde{x}_{i-1}\right) \\
\left(\bar{x}_{i}, \tilde{x}_{i-1}\right) \text { and }\left(\hat{r}_{i}, \hat{x}_{i-1}\right) \\
\left(\hat{r}_{i}, \hat{x}_{i-1}\right)
\end{array}\right\} \Longleftrightarrow \\
F^{i}\left(s_{i} \equiv \bar{x}_{i}, \tilde{x}_{i-1}\right)\left\{\begin{array}{c}
> \\
= \\
<
\end{array}\right\} F^{i}\left(s_{i} \equiv \hat{r}_{i}, \hat{x}_{i-1}\right) .
\end{array}
$$

Making use of the definitions of $F^{i}\left(s_{i} \equiv \bar{x}_{i}, \tilde{x}_{i-1}\right)$ and $F^{i}\left(s_{i} \equiv \hat{r}_{i}, \hat{x}_{i-1}\right)$ it is easy to show

$$
\text { A2: } \quad F^{i}\left(s_{i} \equiv \bar{x}_{i}, \tilde{x}_{i-1}\right)\left\{\begin{array}{c}
> \\
= \\
<
\end{array}\right\} F^{i}\left(s_{i} \equiv \hat{r}_{i}, \hat{x}_{i-1}\right) \Longleftrightarrow \bar{x}_{i}\left\{\begin{array}{l}
< \\
= \\
>
\end{array}\right\} \bar{X}^{i}\left(p_{i}, p_{i-1}\right),
$$

where $\bar{X}^{i}\left(p_{i}, p_{i-1}\right):=\frac{2 k_{i}\left(a_{i} n_{i}-e_{i}-f_{i} p_{i-1}\right)-k_{i}^{2}-2 c_{i} g_{i} p_{i}}{2 c_{i}}$. Next we show

\footnotetext{
${ }^{21}$ We restrict our attention to interior maximizers, in what follows. Boundary solutions may play a role, too, in particular when the focus is on species extinction.
} 
A3: $\quad$ If $\left(p, s^{*}, x^{*}, \bar{x}\right)$ is a short-run ecosystem equilibrium, one has

$$
s_{i}^{*}\left\{\begin{array}{l}
= \\
<
\end{array}\right\} \bar{x}_{i} \Longleftrightarrow p_{i}\left\{\begin{array}{l}
= \\
>
\end{array}\right\} 0 .
$$

Proof of A3: The equivalence of $s_{i}^{*}=\bar{x}_{i}$ and $p_{i}=0$ is straightforward from (4b), (4d) and (4e). It remains to show that $s_{i}^{*}<\bar{x}_{i}$ iff $p_{i}>0$.

Sufficiency. $p_{i}>0$ implies $\bar{x}_{i}=X^{i}\left(p_{-i}, \bar{x}\right)<x_{i}^{*}$. Moreover $s_{i}^{*}=x_{i}^{*}$ via $(4 \mathrm{~d})$. Hence $s_{i}^{*}>\bar{x}_{i}$.

Necessity. $s_{i}^{*}<\bar{x}_{i}$ and $\bar{x}_{i}=X^{i}\left(p_{-i}, \bar{x}\right)$ yields $s_{i}^{*}<X^{i}\left(p_{-i}, \bar{x}\right)$. The equilibrium condition $s_{i}^{*}=x_{i}^{*}=X^{i}(p, \bar{x})$ cannot be secured unless $p_{i}>0$ since $X^{i}(p, \bar{x})$ is strictly declining in $p_{i}$.

We now combine A1 and A2 to obtain

A4: $\quad$ If $\left(p, s^{*}, x^{*}, \bar{x}\right)$ is a short-run ecosystem equilibrium, then

$$
\left(s_{i}^{*}, x_{i-1}^{*}\right)=\left\{\begin{array}{c}
\left(\bar{x}_{i}, \tilde{x}_{i-1}\right) \\
\left(\bar{x}_{i}, \tilde{x}_{i-1}\right) \text { and }\left(\hat{r}_{i}, \hat{x}_{i-1}\right) \\
\left(\hat{r}_{i}, \hat{x}_{i-1}\right)
\end{array}\right\} \Longleftrightarrow \bar{x}_{i}\left\{\begin{array}{l}
< \\
= \\
>
\end{array}\right\} \bar{X}^{i}\left(p_{i}, p_{i-1}\right)
$$

which constitutes the core of Proposition 1. In view of A3 and A4 it is straightforward to specify the relationship between the sign of the price $p_{i}$ and the sign of $\bar{x}_{i}-\bar{X}^{i}\left(p_{i}, p_{i-1}\right)$ as follows:

A5: $\quad$ If $\left(p, s^{*}, x^{*}, \bar{x}\right)$ is a short-run ecosystem equilibrium, then

$$
\begin{gathered}
\bar{x}_{i}\left\{\begin{array}{l}
< \\
= \\
>
\end{array}\right\} \bar{X}^{i}\left(p_{i}, p_{i-1}\right) \quad \Longrightarrow \quad p_{i}\left\{\begin{array}{l}
= \\
= \\
>
\end{array}\right\} 0, \\
p_{i}\left\{\begin{array}{l}
= \\
>
\end{array}\right\} 0 \quad \Longrightarrow \quad \bar{x}_{i}\left\{\begin{array}{c}
\leq \\
>
\end{array}\right\} \bar{X}^{i}\left(p_{i}, p_{i-1}\right) .
\end{gathered}
$$

\section{Appendix B: Characterization of short-run ecosystem equilibria}

\section{Demands and supplies.}

Demands and supplies are listed in Table 6 and 7.

\section{Equilibrium prices.}


For obvious reasons we only need to focus on positive prices. If $p_{i}>0$ in some regime under consideration we equate demand and supply and solve for the price to obtain:

Regime 2.

$$
p_{2}=\frac{a_{3} c_{2} n_{3}-a_{2} c_{3} k_{2} n_{2}-c_{2} e_{3}+c_{3} k_{2}\left(e_{2}+k_{2}\right)}{c_{2}\left(c_{3} g_{2}+f_{3}\right)} .
$$

Regime 3.

$$
\begin{aligned}
& p_{1}=\frac{a_{2} c_{1} n_{2}-a_{1} c_{2} k_{1} n_{1}-c_{1}\left(e_{2}+k_{2}\right)+c_{2} k_{1}\left(e_{1}+k_{1}\right)}{c_{1}\left(c_{2} g_{1}+f_{2}\right)} \\
& p_{2}=\frac{a_{3} c_{2} n_{3}-a_{2} c_{3} k_{2} n_{2}-c_{2} e_{3}+c_{3} k_{2}\left(e_{2}+k_{2}+f_{1} p_{1}\right)}{c_{2}\left(c_{3} g_{2}+f_{3}\right)} .
\end{aligned}
$$

Regime 4.

$$
p_{1}=\frac{a_{2} c_{1} n_{2}-a_{1} c_{2} k_{1} n_{1}-c_{1}\left(e_{2}+k_{2}\right)+c_{2} k_{1}\left(e_{1}+k_{1}\right)}{c_{1}\left(c_{2} g_{1}+f_{2}\right)} .
$$

Regime 5.

$$
\begin{aligned}
& p_{0}=\frac{a_{1} n_{1}-e_{1}-k_{1}-c_{1} \bar{s}_{0}}{f_{1}}, \\
& p_{1}=\frac{a_{2} n_{2}-e_{2}-k_{2}-c_{2} k_{1} \bar{s}_{0}}{c_{2} g_{1}+f_{2}} .
\end{aligned}
$$

Regime 6.

$$
p_{0}=\frac{a_{1} n_{1}-e_{1}-k_{1}-c_{1} \bar{s}_{0}}{f_{1}}
$$

Regime $\%$.

$$
\begin{aligned}
& p_{0}=\frac{a_{1} n_{1}-e_{1}-k_{1}-c_{1} \bar{s}_{0}}{f_{1}} \\
& p_{2}=\frac{a_{3} c_{2} n_{3}-a_{2} c_{3} k_{2} n_{2}-c_{2} e_{3}+c_{3} k_{2}\left(e_{2}+k_{2}\right)}{c_{2}\left(c_{3} g_{2}+f_{3}\right)} .
\end{aligned}
$$

Regime 8.

$$
\begin{aligned}
& p_{0}=\frac{a_{1} n_{1}-e_{1}-k_{1}-c_{1} \bar{s}_{0}}{f_{1}} \\
& p_{1}=\frac{a_{2} n_{2}-e_{2}-k_{2}-c_{2} k_{1} \bar{s}_{0}}{c_{2} g_{1}+f_{2}}, \\
& p_{2}=\frac{a_{3} n_{3}-e_{3}-c_{3} k_{1} k_{2} \bar{s}_{0}-\frac{c_{3} g_{1} k_{2}}{c_{2} g_{1}+f_{2}}\left(a_{2} n_{2}-e_{2}-k_{2}-c_{2} k_{1} \bar{s}_{0}\right)}{c_{3} g_{2}+f_{3}} .
\end{aligned}
$$

\section{Parameters defining the support sets of equilibrium regimes.}

For each regime $j, j=1, \ldots, 8$, Table 2 lists three inequalities defining the support set of populations of that regime. The procedure of how to obtain those inequalities is demonstrated in section 3 for the regimes 1 and 8, resulting in (8) and (12), respectively. The 
calculations for the remaining regimes $2, \ldots, 7$ follow the same methodology and can be obtained from the authors upon request. It suffices here to define the parameters $\sigma_{1}, \ldots, \sigma_{10}$ used in Table 2.

$$
\begin{aligned}
\sigma_{1} & :=\frac{e_{1}+k_{1}+c_{1} \bar{s}_{0}}{a_{1}}, \sigma_{2}:=\frac{a_{1} c_{2} k_{1}}{a_{2} c_{1}}, \sigma_{3}:=\frac{c_{1}\left(e_{2}+k_{2}\right)-c_{2} k_{1}\left(e_{1}+k_{1}\right)}{a_{2} c_{1}}, \\
\sigma_{4} & :=\frac{a_{2} c_{3} k_{2}}{a_{3} c_{2}}, \sigma_{5}:=\frac{c_{2} e_{3}-c_{3} k_{2}\left(e_{2}+k_{2}\right)}{a_{3} c_{2}}, \sigma_{6}:=\frac{a_{2} c_{3} k_{2}}{a_{3} c_{2}}-\frac{a_{2} c_{3} f_{1} k_{2}}{a_{3} c_{2}\left(c_{2} g_{1}+f_{2}\right)}, \\
\sigma_{7} & :=\frac{a_{1} c_{2} c_{3} f_{1} k_{1} k_{2}}{a_{3} c_{1} c_{2}\left(c_{2} g_{1}+f_{2}\right)}, \sigma_{8}:=\frac{c_{2} e_{3}-c_{3} k_{2}\left(e_{2}+k_{2}\right)}{a_{3} c_{2}}-\frac{c_{3} f_{1} k_{2}}{a_{3} c_{2}}\left[\frac{c_{2} k_{1}\left(e_{1}+k_{1}\right)-c_{1}\left(e_{2}+k_{2}\right)}{c_{1}\left(c_{2} g_{1}+f_{2}\right)}\right], \\
\sigma_{9} & :=\frac{e_{2}+k_{2}+c_{2} k_{1} \bar{s}_{0}}{a_{2}}, \sigma_{10}:=\frac{\left(c_{2} g_{1}+f_{2}\right)\left(e_{3}+k_{3}+c_{3} k_{1} k_{2} \bar{s}_{0}\right)-c_{3} g_{1} k_{2}\left(e_{2}+k_{2}+c_{2} k_{2} \bar{s}_{0}\right)}{a_{3}\left(c_{2} g_{1}+f_{2}\right)} .
\end{aligned}
$$

\section{Appendix C: Population growth depending on equilibrium regimes}

Since equilibrium allocations are regime-specific, the population changes (1) associated to those equilibrium allocations are regime-specific, too. To determine these changes, we start with regime 1 as characterized in Section 3. Recall, that in view of (1), (3), (4) and Propositions 1 and 2 we have

$$
\begin{aligned}
& \dot{n}_{i}=\left(a_{i} n_{i}-e_{i}-f_{i} p_{i-1}\right) x_{i-1}-\frac{c_{i}}{2} x_{i-1}^{2}-\bar{x}_{i}-d_{i} n_{i} \quad \text { for } i=1,2, \\
& \dot{n}_{3}=\left(a_{3} n_{3}-e_{3}-f_{3} p_{2}\right) x_{2}-\frac{c_{3}}{2} x_{2}^{2}-d_{3} n_{3} .
\end{aligned}
$$

From (9) we invoke $x_{i-1}^{*}=\left(a_{i} n_{i}-e_{i}\right) / c_{i}$ for $i=1,2,3$ and $\bar{x}_{i}=\bar{X}^{i}\left(p_{-i}, \bar{x}, n\right)=\left(a_{i+1} n_{i+1}-\right.$ $\left.e_{i+1}\right) / c_{i+1}$ for $i=1,2$. We insert $\bar{x}_{i}$ and $x_{i-1}^{*}$ into (13) and set $p_{i}=0$ for $i=0,1,2$ to obtain, after some rearrangement of terms,${ }^{22}$

$$
\begin{aligned}
& \dot{n}_{1}=\lambda_{1} n_{1}^{2}-\lambda_{2} n_{1}-\lambda_{3} n_{2}+\lambda_{4}, \\
& \dot{n}_{2}=\mu_{1} n_{2}^{2}-\mu_{2} n_{2}-\mu_{3} n_{3}+\mu_{4}, \\
& \dot{n}_{3}=\rho_{1} n_{3}^{2}-\rho_{2} n_{3}+\rho_{3} .
\end{aligned}
$$

The equations (40) constitute a system of differential equations that completely describe the population dynamics for all $\left(n_{1}, n_{2}, n_{3}\right) \in N_{1}$.

To fix our ideas we consider species 3 and suppose that $\dot{n}_{3}>0$. We then infer from (41c) that $\dot{n}_{3}>0$ for all future periods implying that $n_{3}$ will eventually violate (7). In other words, we are bound to leave regime 1 at some future date. The signs of $\dot{n}_{1}$ and $\dot{n}_{2}$ cannot be as easily determined as the sign of $\dot{n}_{3}$ because for $i=1,2$ the time path of $n_{i}$ depends on the time path of $n_{i+1}$. For the time being it suffices to make the following observation: Suppose the ecosystem is endowed with $\left(n_{1}, n_{2}, n_{3}\right) \in N_{1}$ in some initial (short) period

\footnotetext{
${ }^{22}$ The parameters $\lambda, \mu$ and $\rho$ used in the subsequent analysis are defined at the end of Appendix C.
} 
such that the associated equilibrium belongs to regime 1 . Then the population dynamics unfolding in subsequent periods are described by (40). Populations may shrink ${ }^{23}$ or grow and if a population grows sufficiently the price of its biomass becomes eventually positive which is tantamount to saying that the prevailing vector of populations is no longer in $N_{1}$ and that, as a consequence, the population dynamics are no longer adequately described by $(40)$.

At the very point in time when $\left(n_{1}, n_{2}, n_{3}\right)$ leaves the set $N_{1}$ the vector $\left(n_{1}, n_{2}, n_{3}\right)$ is bound to enter the support set $N_{j}$ of some other regime $j \neq 1$. Unless some species gets extinct, the ecosystem is bound to leave regime 1 heading either ${ }^{24}$ for regime 2,4 or 6. Since it is by no means clear, a priori, which the sequence of regimes is passed by the ecosystem in the course of time, all regimes need to be fully specified in terms of both their equilibrium properties and their specific population dynamics.

To spell out the population dynamics prevailing in regime 8 we can take over equation (40b) but we need to substitute (40a) by

$$
\dot{n}_{i}=\left(a_{i} n_{i}-e_{i}-k_{i}-f_{i} p_{i-1}\right) x_{i-1}-\frac{c_{i}}{2} x_{i-1}^{2}-g_{i} p_{i}-d_{i} n_{i} \quad \text { for } i=1,2 .
$$

Next we insert the appropriate prices from (11) and the biomass demands from (13) into (40b) and (42), respectively. Following simple but tedious calculations we obtain

$$
\begin{aligned}
& \dot{n}_{1}=-d_{1} n_{1}-\lambda_{7} n_{2}+\lambda_{9} \\
& \dot{n}_{2}=\mu_{8} n_{2}^{2}-\mu_{19} n_{2}-\mu_{6} n_{3}+\mu_{20}, \\
& \dot{n}_{3}=\rho_{4} n_{3}^{2}-\rho_{19} n_{3}+\rho_{20} n_{2}^{2}-\rho_{21} n_{2}+\rho_{22} n_{3} n_{2}+\rho_{23} .
\end{aligned}
$$

Comparing the differential equations (40) and (42) reveals that the equation of motion of $n_{1}$ is distinctly different across both regimes. For $n_{2}$ the equation of motion is qualitatively the same in both regimes but in case of species 3 we observe a dependence of $\dot{n}_{3}$ on $n_{2}$ in regime 8 which was absent in regime 1.

\section{Parameters defining the differential equations.}

$$
\begin{aligned}
\lambda_{1} & :=\frac{a_{1}^{2}}{2 c_{1}}, \lambda_{2}:=d_{1}+\frac{a_{1} e_{1}}{c_{1}}, \lambda_{3}:=\frac{a_{2}}{c_{2}}, \lambda_{4}:=\frac{e_{1}^{2}}{2 c_{1}}+\frac{e_{2}}{c_{2}}, \lambda_{5}:=\frac{e_{1}^{2}}{2 c_{1}}+\frac{e_{2}+k_{2}}{c_{2}}, \\
\lambda_{6} & :=d_{1}+\frac{a_{1} e_{1}\left(c_{2} g_{1}+f_{2}\right)+a_{1} f_{2} k_{1}}{c_{1}\left(c_{2} g_{1}+f_{2}\right)}, \lambda_{7}:=\frac{a_{2} g_{1}}{c_{2} g_{1}+f_{2}}, \\
\lambda_{8} & :=\frac{e_{1}\left(c_{2} g_{1}+f_{2}\right)+\left(2 e_{1} k_{1}+k_{1}^{2}\right) f_{2}-c_{2} g_{1} k_{1}^{2}+2 c_{1} g_{1}\left(e_{2}+k_{2}\right)}{2 c_{1}\left(c_{2} g_{1}+f_{2}\right)}
\end{aligned}
$$

\footnotetext{
${ }^{23}$ If $\dot{n}_{i}<0$ for some $i$ at some date $t$ it may happen that $\dot{n}_{i}<0$ for all future periods such that species $i$ will not survive in the ecosystem under consideration.

${ }^{24}$ The ecosystem may even switch from regime 1 to regime 3,5 or 8 but the simultaneous passing of more than one boundary line is extremely unlikely.
} 


$$
\begin{aligned}
& \lambda_{9}:=\frac{g_{1}\left(e_{2}+k_{2}+c_{2} k_{1} \bar{s}_{0}\right)}{c_{2} g_{1}+f_{2}}+\frac{c_{1} \bar{s}_{0}^{2}}{2}, \lambda_{10}:=\bar{s}_{0}\left(k_{1}+\frac{c_{1} \bar{s}_{0}}{2}\right)+\frac{e_{2}}{c_{2}}, \\
& \lambda_{11}:=\bar{s}_{0}\left(k_{1}+\frac{c_{1} \bar{s}_{0}}{2}\right)+\frac{e_{2}+k_{2}}{c_{2}} . \\
& \mu_{1}:=\frac{a_{2}^{2}}{2 c_{2}}, \mu_{2}:=d_{2}+\frac{a_{2} e_{2}}{c_{2}}, \mu_{3}:=\frac{a_{3}}{c_{3}} \mu_{4}:=\frac{e_{2}^{2}}{2 c_{2}}+\frac{e_{3}}{c_{3}}, \\
& \mu_{5}:=d_{2}+\frac{a_{2} f_{3}\left(e_{2}+k_{2}\right)+a_{2} c_{3} e_{2} g_{2}}{c_{2}\left(c_{3} g_{2}+f_{3}\right)}, \mu_{6}:=\frac{a_{3} g_{2}}{c_{3} g_{2}+f_{3}}, \\
& \mu_{7}:=\frac{\left(e_{2}+k_{2}\right)^{2} f_{3}+c_{3} g_{2}\left(e_{2}^{2}-k_{2}^{2}\right)+2 c_{2} e_{3} g_{2}}{c_{2}\left(c_{3} g_{2}+f_{3}\right)}, \mu_{8}:=\frac{a_{2}^{2} c_{2} g_{1}^{2}}{2\left(c_{2} g_{1}+f_{2}\right)^{2}}, \\
& \mu_{9}:=d_{2}+\frac{a_{2} g_{1} \alpha_{1}}{c_{1}\left(c_{2} g_{1}+f_{2}\right)^{2}}-\frac{a_{2} c_{3} g_{2} k_{2}}{c_{2}\left(c_{3} g_{2}+f_{3}\right)}+\frac{a_{2} c_{3} f_{1} g_{2} k_{2}}{c_{2}\left(c_{2} g_{1}+f_{2}\right)\left(c_{3} g_{2}+f_{3}\right)}, \\
& \mu_{10}:=\frac{a_{1}^{2} c_{2} f_{2}^{2} k_{1}^{2}}{2 c_{1}^{2}\left(c_{2} g_{1}+f_{2}\right)^{2}}, \mu_{11}:=\frac{a_{1} f_{2} k_{1} \alpha_{1}}{c_{1}^{2}\left(c_{2} g_{1}+f_{2}\right)^{2}}-\frac{a_{1} g_{2} k_{1}}{c_{1}\left(c_{2} g_{1}+f_{2}\right)\left(c_{3} g_{2}+f_{3}\right)}, \\
& \mu_{12}:=\frac{a_{1} a_{2} c_{2} f_{2} g_{1} k_{1}}{c_{1}\left(c_{2} g_{1}+f_{2}\right)^{2}} \\
& \mu_{13}:=\frac{\alpha_{1}^{2}}{2 c_{1}^{2} c_{2}\left(c_{2} g_{1}+f_{2}\right)^{2}}+\frac{c_{2} e_{3} g_{2}-c_{3} g_{2} k_{2}\left[e_{2}+k_{2}+\frac{f_{1}\left(c_{2} k_{1}\left(e_{1}+k_{1}\right)-c_{1}\left(e_{2}+k_{2}\right)\right)}{c_{1}\left(c_{2} g_{1}+f_{2}\right)}\right]}{c_{2}\left(c_{3} g_{2}+f_{3}\right)}, \\
& \mu_{14}:=d_{2}+\frac{g_{1} \alpha_{2}}{c_{1}\left(c_{2} g_{1}+f_{2}\right)^{2}}, \mu_{15}:=\frac{a_{1} f_{2} k_{1} \alpha_{2}}{c_{1}^{2}\left(c_{2} g_{1}+f_{2}\right)^{2}}, \mu_{16}:=\frac{\alpha_{2}^{2}}{2 c_{2} c_{1}^{2}\left(c_{2} g_{1}+f_{2}\right)^{2}}-\frac{k_{2}^{2}}{2 c_{2}}+\frac{e_{3}}{c_{3}}, \\
& \mu_{17}:=d_{2}-\frac{a_{2} g_{1} \alpha_{3}}{\left(c_{2} g_{1}+f_{2}\right)^{2}}, \mu_{18}:=\frac{1}{2 c_{2}}\left[\frac{\alpha_{3}^{2}}{\left(c_{2} g_{1}+f_{2}\right)^{2}}-k_{2}^{2}\right]+\frac{e_{3}}{c_{3}}, \\
& \mu_{19}:=d_{2}+\frac{a_{2} g_{1} \alpha_{4}}{\left(c_{2} g_{1}+f_{2}\right)^{2}}-\frac{a_{2} c_{3} g_{1} g_{2} k_{2}}{\left(c_{2} g_{1}+f_{2}\right)\left(c_{3} g_{2}+f_{3}\right)}, \\
& \mu_{20}:=\frac{\alpha_{4}^{2}}{2 c_{2}\left(c_{2} g_{1}+f_{2}\right)^{2}}+\frac{e_{3} g_{2}+c_{3} g_{2} k_{1} k_{2} \bar{s}_{0}-\frac{c_{3} g_{1} g_{2} k_{1}}{c_{2} g_{1}+f_{2}}\left(e_{2}+k_{2}+c_{2} k_{1} \bar{s}_{0}\right)}{c_{3} g_{2}+f_{3}},
\end{aligned}
$$

where $\alpha_{1}:=c_{1} c_{2} g_{1}\left(e_{2}+k_{2}\right)+c_{2} f_{2} k_{1}\left(e_{1}+k_{1}\right), \alpha_{2}:=c_{1} c_{2} e_{2} g_{1}+c_{2} f_{2} k_{1}\left(e_{1}+k_{1}\right)-c_{1} f_{2} k_{2}$, $\alpha_{3}:=f_{2} k_{2}+c_{2} f_{2} k_{1} \bar{s}_{0}-c_{2} e_{2} g_{1}, \alpha_{4}:=c_{2} g_{1}\left(e_{2}+k_{2}\right)-c_{2} f_{2} k_{1} \bar{s}_{0}$.

$$
\begin{aligned}
& \rho_{1}:=\frac{a_{3}^{2}}{2 c_{3}}, \rho_{2}:=d_{3}+\frac{a_{3} e_{3}}{c_{3}}, \rho_{3}:=\frac{e_{3}^{2}}{2 c_{3}} \rho_{4}:=\frac{a_{3}^{2} c_{3} g_{2}^{2}}{2\left(c_{3} g_{2}+f_{3}\right)^{2}}, \\
& \rho_{5}:=d_{3}+\frac{a_{3} g_{2} \alpha_{5}}{c_{2}\left(c_{3} g_{2}+f_{3}\right)^{2}}, \rho_{6}:=\frac{a_{2}^{2} c_{3} f_{3}^{2} k_{2}^{2}}{2 c_{2}^{2}\left(c_{3} g_{2}+f_{3}\right)^{2}}, \rho_{7}:=\frac{a_{2} f_{3} k_{2} \alpha_{5}}{c_{2}^{2}\left(c_{3} g_{2}+f_{3}\right)^{2}}, \\
& \rho_{8}:=\frac{a_{2} a_{3} c_{3} f_{3} g_{2} k_{2}}{c_{2}\left(c_{3} g_{2}+f_{3}\right)^{2}}, \rho_{9}:=\frac{\alpha_{5}^{2}}{2 c_{3} c_{2}^{2}\left(c_{3} g_{2}+f_{3}\right)^{2}}, \rho_{10}:=d_{3}+\frac{a_{3} g_{2} \alpha_{6}}{c_{3} g_{2}+f_{3}}, \\
& \rho_{11}:= \frac{\left(a_{2} c_{3} f_{3} k_{2}-\frac{a_{2} c_{3} f_{1} f_{3} k_{2}}{c_{2} g_{1}+f_{2}}\right)^{2}}{2 c_{3} c_{2}^{2}\left(c_{3} g_{2}+f_{3}\right)^{2}}, \rho_{12}:=\alpha_{6} \frac{a_{2} f_{3} k_{2}-\frac{a_{2} f_{1} f_{3} k_{2}}{c_{2} g_{1}+f_{2}}}{c_{2}\left(c_{3} g_{2}+f_{3}\right)}, \\
& \rho_{13}:=\frac{a_{1}^{2} c_{3} f_{1}^{2} f_{3}^{2} k_{1}^{2} k_{2}^{2}}{2 c_{1}^{2}\left(c_{2} g_{1}+f_{2}\right)^{2}\left(c_{3} g_{2}+f_{3}\right)^{2}}, \rho_{14}:=\alpha_{6} \frac{a_{1} f_{1} f_{3} k_{1} k_{2}}{c_{1}\left(c_{2} g_{1}+f_{2}\right)\left(c_{3} g_{2}+f_{3}\right)}, \\
& \rho_{15}:=a_{3} g_{2} \frac{a_{2} c_{3} f_{3} k_{2}-\frac{a_{2} c_{3} f_{1} f_{3} k_{2}}{c_{2} g_{1}+f_{2}}}{c_{3} g_{2}+f_{3}}, \rho_{16}:=\frac{a_{1} a_{3} c_{2} c_{3} g_{2} f_{1} f_{3} k_{1} k_{2}}{c_{1}\left(c_{2} g_{1}+f_{2}\right)\left(c_{3} g_{2}+f_{3}\right)}, \\
& \rho_{17}:=\left(a_{2} c_{3} f_{3} k_{2}-\frac{a_{2} c_{3} f_{1} f_{3} k_{2}}{c_{2} g_{1}+f_{2}}\right)\left(\frac{a_{1} f_{1} f_{3} k_{1} k_{2}}{c_{1}\left(c_{2} g_{1}+f_{2}\right)\left(c_{3} g_{2}+f_{3}\right)}\right), \rho_{18}:=\frac{\alpha_{6}^{2}}{2 c_{3} c_{2}^{2}\left(c_{3} g_{2}+f_{3}\right)},
\end{aligned}
$$




$$
\begin{aligned}
\rho_{19}:= & d_{3}+\frac{a_{3} g_{2} \alpha_{7}}{\left(c_{3} g_{2}+f_{3}\right)^{2}}, \rho_{20}:=\frac{a_{2}^{2} c_{3} f_{3}^{2} g_{1}^{2} k_{2}^{2}}{2\left(c_{3} g_{2}+f_{3}\right)^{2}\left(c_{2} g_{1}+f_{2}\right)^{2}}, \rho_{21}:=\frac{a_{2} f_{3} g_{1} k_{2} \alpha_{7}}{\left(c_{3} g_{2}+f_{3}\right)^{2}\left(c_{2} g_{1}+f_{2}\right)}, \\
\rho_{22}:= & \frac{a_{2} a_{3} c_{3} f_{3} g_{1} g_{2} k_{2}}{\left(c_{3} g_{2}+f_{3}\right)^{2}\left(c_{2} g_{1}+f_{2}\right)}, \rho_{23}:=\frac{\alpha_{7}^{2}}{2 c_{3}\left(c_{3} g_{2}+f_{3}\right)^{2}}, \\
& \text { where } \alpha_{5}:=c_{2} c_{3} e_{3} g_{2}+c_{3} f_{3} k_{2}\left(e_{2}+k_{2}\right), \\
\alpha_{6}:= & c_{2} c_{3} e_{3} g_{2}+c_{3} f_{3} k_{2}\left(e_{2}+k_{2}\right)-\frac{c_{3} f_{1} f_{3} k_{2}}{c_{1}\left(c_{2} g_{1}+f_{2}\right)}\left[c_{1}\left(e_{2}+k_{2}\right)-c_{2} k_{1}\left(e_{1}+k_{1}\right)\right], \\
\alpha_{7}:= & c_{3} e_{3} g_{2}-c_{3} f_{3} k_{1} k_{2} \bar{s}_{0}+\frac{c_{3} f_{3} g_{1} k_{2}}{c_{2} g_{1}+f_{2}}\left(e_{2}+k_{2}+c_{2} k_{1} \bar{s}_{0}\right) .
\end{aligned}
$$

\section{Numerical values of parameters used in the examples of Section 5.}

(a) Parameter values for the examples 1 and 2

$$
\begin{aligned}
& a_{1}=2, \quad a_{2}=4, \quad a_{3}=4 \quad c_{1}=10, \quad c_{2}=50, \quad c_{3}=60, \quad d_{1}=2, \quad d_{2}=2, \\
& d_{3}=2, \quad e_{1}=10, \quad e_{2}=10, \quad e_{3}=10, \quad f_{1}=1, \quad f_{2}=10, \quad f_{3}=20, \quad g_{1}=1, \\
& g_{2}=1, \quad k_{1}=10, \quad k_{2}=100, \quad \bar{s}_{0}=500 \text {. }
\end{aligned}
$$

(b) Parameter values for example 3

As in (a) except that $g_{2}=1$ is replaced by $g_{2}=0$.

(c) Parameter values for example 4

$$
\begin{aligned}
& a_{1}=1, \quad a_{2}=1, \quad a_{3}=1 \quad c_{1}=1, \quad c_{2}=1, \quad c_{3}=0.1, \quad d_{1}=1, \quad d_{2}=1, \\
& d_{3}=1, \quad e_{1}=1, \quad e_{2}=1, \quad e_{3}=10, \quad f_{1}=1, \quad f_{2}=1, \quad f_{3}=1 \quad g_{1}=0, \\
& g_{2}=1, \quad k_{1}=1, \quad k_{2}=1, \quad \bar{s}_{0}=0.5 \quad \bar{n}_{1}=10 \quad n_{2}(0)=1 \quad n_{3}(0)=0.1 .
\end{aligned}
$$

(d) Parameter values for example 5

$$
\begin{aligned}
& a_{1}=3, \quad a_{2}=6, \quad a_{3}=4 \quad c_{1}=10, \quad c_{2}=2, \quad c_{3}=2, \quad d_{1}=10, \\
& d_{2}=100, \quad d_{3}=2, \quad e_{1}=50, \quad e_{2}=50, \quad e_{3}=100, \quad f_{1}=5, \quad f_{2}=5, \\
& f_{3}=0.2, \quad g_{1}=20, \quad g_{2}=20, \quad k_{1}=0.5, \quad k_{2}=10, \quad \bar{s}_{0}=100 \quad \bar{n}_{3}=200 \\
& n_{1}(0)=50 \quad n_{2}(0)=0.5 \text {. }
\end{aligned}
$$

(e) Parameter values for example 6

$$
\begin{aligned}
& a_{1}=1, \quad a_{2}=1, \quad a_{3}=1 \quad c_{1}=1, \quad c_{2}=1, \quad c_{3}=1, \quad d_{1}=0.5, \\
& d_{2}=1, \quad d_{3}=10, \quad e_{1}=1, \quad e_{2}=5, \quad e_{3}=0, \quad f_{1}=1.5, \quad f_{2}=5, \\
& f_{3}=10, \quad g_{1}=1, \quad g_{2}=1, \quad k_{1}=1, \quad k_{2}=0.01, \quad \bar{s}_{0}=20 \quad n_{1}(0)=300 \\
& n_{2}(0)=100 \quad n_{3}(0)=150 \text {. }
\end{aligned}
$$

The specification $g_{2}=0$ in (b) is an auxiliary assumption that needs to complement the assumption of keeping population 2 constant in (b) while the population of the predator species 3 is endogenous. A growing predator population drives up the price for prey biomass. If the price elasticity of supply were positive and non-vanishing an ever increasing intake of prey biomass would induce unlimited growth of the predator species which is only due, however, to the simplifying assumption that the population of the prey species is constant. 


\begin{tabular}{|c|c|c|c|c|}
\hline & $\mathrm{R} 1$ & $\mathrm{R} 2$ & $\mathrm{R} 3$ & $\mathrm{R} 4$ \\
\hline$p_{0}$ & 0 & 0 & 0 & 0 \\
\hline$p_{1}$ & 0 & 0 & 1 & 1 \\
\hline$p_{2}$ & 0 & 1 & 1 & 0 \\
\hline & $\bar{x}_{1}<\bar{X}_{1}$ & $\bar{x}_{1}<\bar{X}_{1}$ & $\bar{x}_{1} \geq \bar{X}_{1}$ & $\bar{x}_{1} \geq \bar{X}_{1}$ \\
\hline & $\bar{x}_{2}<\bar{X}_{2}$ & $\bar{x}_{2} \geq \bar{X}_{2}$ & $\bar{x}_{2} \geq \bar{X}_{2}$ & $\bar{x}_{2}<\bar{X}_{2}$ \\
\hline$x_{0}$ & $\frac{a_{1} n_{1}-e_{1}}{c_{1}}$ & $\frac{a_{1} n_{1}-e_{1}}{c_{1}}$ & $\frac{a_{1} n_{1}-e_{1}-k_{1}}{c_{1}}$ & $\frac{a_{1} n_{1}-e_{1}-k_{1}}{c_{1}}$ \\
\hline$x_{1}$ & $\frac{a_{2} n_{2}-e_{2}}{c_{2}}$ & $\frac{a_{2} n_{2}-e_{2}-k_{2}}{c_{2}}$ & $\frac{a_{2} n_{2}-e_{2}-k_{2}-f_{2} p_{1}}{c_{2}}$ & $\frac{a_{2} n_{2}-e_{2}-k_{2}-f_{2} p_{1}}{c_{2}}$ \\
\hline$x_{2}$ & $\frac{a_{3} n_{3}-e_{3}}{c_{3}}$ & $\frac{a_{3} n_{3}-e_{3}-f_{3} p_{2}}{c_{3}}$ & $\frac{a_{3} n_{3}-e_{3}-f_{3} p_{2}}{c_{3}}$ & $\frac{a_{3} n_{3}-e_{3}}{c_{3}}$ \\
\hline$s_{1}$ & $\frac{a_{2} n_{2}-e_{2}}{c_{2}}$ & $\frac{a_{2} n_{2}-e_{2}-k_{2}}{c_{2}}$ & $g_{1} p_{1}+k_{1} x_{0}$ & $g_{1} p_{1}+k_{1} x_{0}$ \\
\hline$s_{2}$ & $\frac{a_{3} n_{3}-e_{3}}{c_{3}}$ & $g_{2} p_{2}+k_{2} x_{1}$ & $g_{2} p_{2}+k_{2} x_{1}$ & $\frac{a_{3} n_{3}-e_{3}}{c_{3}}$ \\
\hline $\bar{x}_{1}$ & $\frac{a_{2} n_{2}-e_{2}}{c_{2}}$ & $\frac{a_{2} n_{2}-e_{2}-k_{2}}{c_{2}}$ & $\frac{a_{2} n_{2}-e_{2}-k_{2}}{c_{2}}$ & $\frac{a_{2} n_{2}-e_{2}}{c_{2}}$ \\
\hline $\bar{x}_{2}$ & $\frac{a_{3} n_{3}-e_{3}}{c_{3}}$ & $\frac{a_{3} n_{3}-e_{3}}{c_{3}}$ & $\frac{a_{3} n_{3}-e_{3}}{c_{3}}$ & $\frac{a_{3} n_{3}-e_{3}}{c_{3}}$ \\
\hline $\bar{X}_{1}$ & $\frac{\frac{k_{1}\left(2 a_{1} n_{1}-2 e_{1}-k_{1}\right)}{2 c_{1}}}{}$ & $\begin{array}{c}\frac{k_{1}\left(2 a_{1} n_{1}-2 e_{1}-k_{1}\right)}{2 c_{1}} \\
\end{array}$ & $\frac{k_{1}\left(2 a_{1} n_{1}-2 e_{1}-k_{1}\right)}{2 c_{1}}$ & $\frac{k_{1}\left(2 a_{1} n_{1}-2 e_{1}-k_{1}\right)}{2 c_{1}}$ \\
\hline $\bar{X}_{2}$ & $\frac{k_{2}\left(2 a_{2} n_{2}-2 e_{2}-k_{2}\right)}{2 c_{2}}$ & $\frac{k_{2}\left(2 a_{2} n_{2}-2 e_{2}-k_{2}\right)}{2 c_{2}}$ & $\frac{k_{2}\left(2 a_{2} n_{2}-2 e_{2}-2 f_{2} p_{1}-k_{2}\right)}{2 c_{2}}$ & $\frac{k_{2}\left(2 a_{2} n_{2}-2 e_{2}-2 f_{2} p_{1}-k_{2}\right)}{2 c_{2}}$ \\
\hline
\end{tabular}

Table 6: Demands, supplies and supply constraints in regimes $1-4$ 


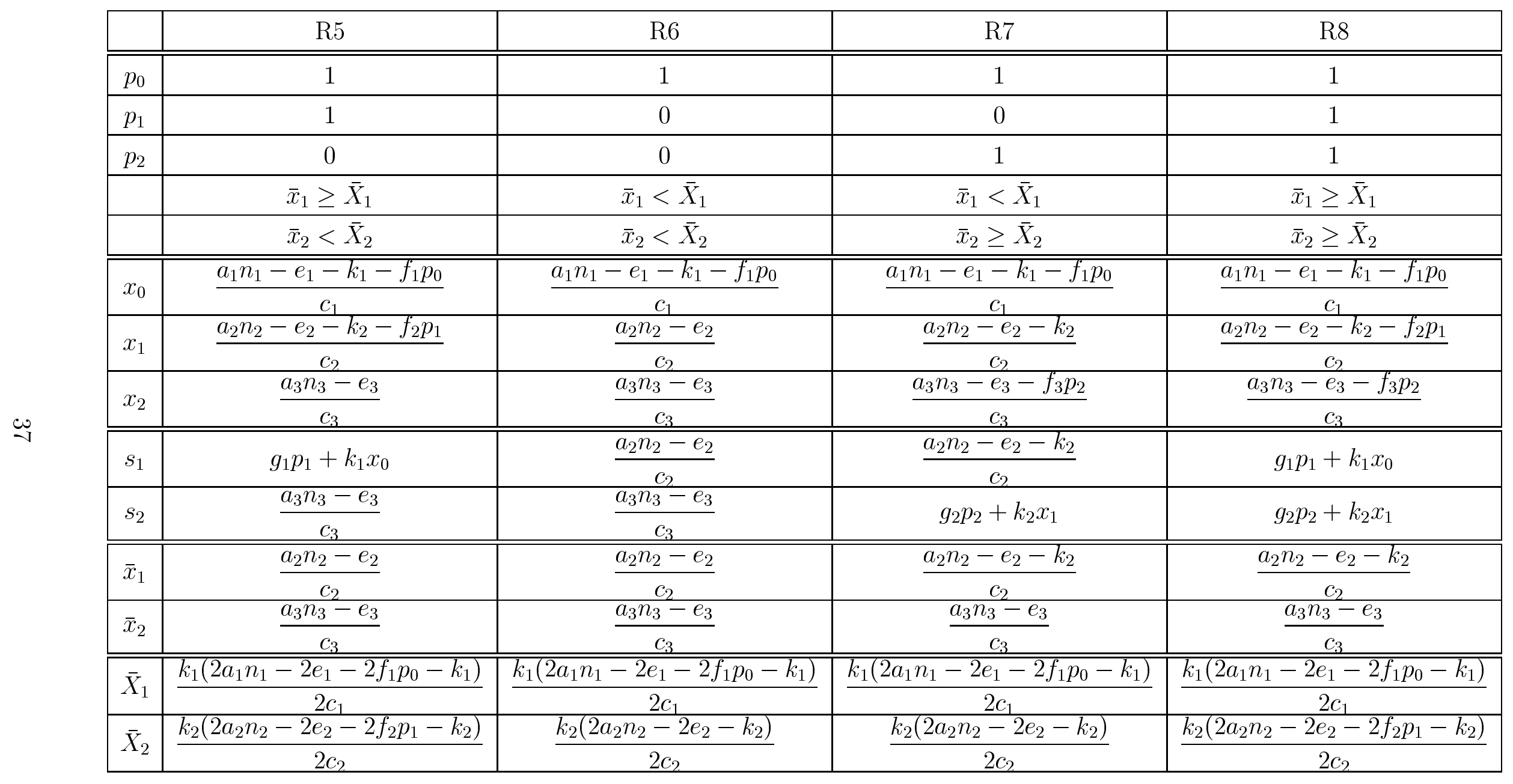

Table 7: Demands, supplies and supply constraints in regimes $5-8$ 